\title{
Supporting Information for \\ Tailoring the Gibbs Free Energy of the Nitroxide Exchange Reaction: Substituent and Solvent Effects
}

\author{
David Sulzer and Angela Bihlmeier* \\ Institute of Physical Chemistry, \\ Karlsruhe Institute of Technology (KIT), \\ Kaiserstr. 12, 76131 Karlsruhe, Germany \\ *E-mail: angela.bihlmeier@kit.edu
}

\section{Contents}

1 Computational Details $\quad$ S2

1.1 Conformational Search . . . . . . . . . . . . . . . . . . . . . S2

1.2 Geometry Optimizations . . . . . . . . . . . . . . . . . . . S2

1.3 Single-Point Energy Calculations . . . . . . . . . . . . . . . . . S2

1.4 Gibbs Free Energies of Solvation . . . . . . . . . . . . . . . . . S3

2 Electronic Energies for the Dissociation and Exchange Reaction S5

2.1 Comparison of $\operatorname{CCSD}(\mathrm{T}) / \mathrm{CBS}$ and $\operatorname{CCSD}(\mathrm{T}) / \mathrm{TZVPP} \ldots \ldots \ldots \ldots$. . . . . . . 5

2.2 Performance of Selected Density Functionals Using the QZVP Basis Set . . . . . S6

3 Cartesian Atomic Coordinates of all Investigated Compounds $\quad$ S7

3.1 Nitroxide Radical Species . . . . . . . . . . . . . . . . . . . . . S7

3.2 Carbon Radical Species . . . . . . . . . . . . . . . . . . . S13

3.3 Alkoxyamines . . . . . . . . . . . . . . . . . . . . . . S14 


\section{Computational Details}

We used the TURBOMOLE program package for all quantum chemical calculations and the COSMOtherm software for the computation of the Gibbs free energies of solvation. If not mentioned otherwise, either the Karlsruhe orbital basis sets of type def2 or the correlationconsistent orbital basis sets of type cc-pVXZ were used.

\subsection{Conformational Search}

A systematic conformational search was performed for all investigated molecules. While the structures of TEMPO (1), TMIO (2), Ben (a), PhEt (b), and Cum (c) are straightforward, several conformational issues have to be taken care of regarding the other radicals and also the alkoxyamines. For the monosubstituted compounds 3-5, the substitution can be done either in axial or in equatorial position and the Et and iPr groups can adapt three orientations depending on the rotation angle. This leads to a total of 6 conformers for $\mathbf{3}$ and $\mathbf{4}$ and 2 conformers for 5. For the tetrasubstituted compounds $\mathbf{6}-\mathbf{8}$, the three possible orientations of the Et and $\mathrm{iPr}$ groups lead to 81 conformers each. In the alkoxyamine structures $\mathbf{1 a}-\mathbf{2} \mathbf{c}$ and $\mathbf{3 b} \mathbf{b} \mathbf{8} \mathbf{b}$, additional aspects, like the rotation about the $\mathrm{NO}-\mathrm{C}$ bond, have to be taken into account. The geometries of all conformers were first optimized at the BP86/SVP level of theory. Only structures within a given energy threshold above the most stable conformer were reoptimized at the B3LYP/TZVP level of theory. Based on these results, the lowest energy conformer for each compound was identified and used in the course of this work.

\subsection{Geometry Optimizations}

Geometry optimizations for all conformers were performed at the BP86/SVP level of theory using the RI approximation for the evaluation of the Coulomb integrals, a quadrature grid of type m3 for the numerical integration of the exchange-correlation contribution and standard convergence criteria (SCF energy: $10^{-6}$ hartree, gradient: $10^{-3}$ hartree/bohr). Reoptimizations of low energy structures were done at the B3LYP/TZVP level of theory using fine quadrature grids (m5) and tight convergence criteria (SCF energy: $10^{-8}$ hartree, gradient: $10^{-5}$ hartree/bohr and inclusion of derivatives of quadrature weights). Force constants and vibrational frequencies were computed in order to ensure that the structures represent minima on the potential energy surface. The B3LYP/TZVP frequencies were also used for the evaluation of the vibrational partition function.

\subsection{Single-Point Energy Calculations}

The B3LYP/TZVP geometries were used for single-point energy calculations at various levels of theory.

Computations for the benchmark value:

- HF calculations were performed with the cc-pVXZ basis sets $(X=\mathrm{Q}, 5,6)$ using tight convergence criteria (SCF energy: $10^{-8}$ hartree, one-electron density: $10^{-7}$ ).

- $\operatorname{CCSD}(\mathrm{T})$ calculations were carried out with the cc-pVXZ basis sets $(X=5,6)$ or the TZVPP basis set in a two-step procedure. The HF calculation was performed using tight convergence criteria (SCF energy: $10^{-8}$ hartree, one-electron density: $10^{-7}$ ). The subsequent $\operatorname{CCSD}(\mathrm{T})$ calculation was done using the frozen core approximation and the RI approximation for the evaluation of the required four-center integrals. Tight convergence criteria for the iterative solution of the coupled cluster equations were employed (CC energy: $10^{-8}$ hartree, vector function: $10^{-8}$ hartree). 
- MP2 calculations with the TZVPP basis set were obtained automatically as a byproduct of the $\operatorname{CCSD}(\mathrm{T})$ calculations described above.

- MP2-F12 calculations were carried out with the cc-pVTZ-F12 basis set. The procedure involves two steps. The HF calculation was performed using tight convergence criteria (SCF energy: $10^{-8}$ hartree, one-electron density: $10^{-7}$ ). The subsequent MP2-F12 calculation was done using the frozen core approximation and the RI approximation for the evaluation of the required four-center integrals in the MP2 and the F12 part. For the F12 part we used ansatz 2, approximation B, and the core Hamiltonian plus Coulomb term for the commutator approximation. The fixed amplitudes method and a Slater-type correlation factor $(\gamma=1.01 / \mathrm{bohr})$ was employed. Further, the RI approximation for the Coulomb and exchange parts and the RI approximation for the three- and four-electron integrals intrinsic to the F12 method were used.

Computations with density functional based methods:

- PBE and TPSS calculations were performed with the TZVP and QZVP basis sets using the RI approximation for the evaluation of the Coulomb integrals, fine quadrature grids (m5) and tight convergence criteria (SCF energy: $10^{-8}$ hartree).

- B3LYP and M06-2X calculations were performed with the TZVP and QZVP basis sets using fine quadrature grids (m5) and tight convergence criteria (SCF energy: $10^{-8}$ hartree).

- B3LYP-D3 calculations were performed with the TZVP basis set and the same criteria as mentioned for the B3LYP calculations. Additionally, the third version of the dispersion correction was employed in combination with the Becke-Johnson damping.

- B2PLYP calculations were carried out with the QZVP basis set and involve a two-step procedure. The DFT calculation was performed using the RI approximation for the evaluation of the Coulomb integrals, fine quadrature grids (m5) and tight convergence criteria (SCF energy: $10^{-8}$ hartree, one-electron density: $10^{-8}$ ). The subsequent MP2-like calculation was done using the frozen core approximation and the RI approximation for the evaluation of the required four-center integrals.

- RPA calculations were carried out with the QZVP basis set and either PBE or TPSS orbitals as input. The procedure involves two steps. The DFT calculation was performed using the RI approximation for the evaluation of the Coulomb integrals, fine quadrature grids (m5) and tight convergence criteria (SCF energy: $10^{-8}$ hartree, one-electron density: $10^{-8}$ ). The subsequent RPA calculation was done using the frozen core approximation and the RI approximation for the evaluation of the required four-center integrals. The number of points for the complex frequency integration was set to 60 .

\subsection{Gibbs Free Energies of Solvation}

The Gibbs free energies of solvation were evaluated according to COSMO-RS theory using the BP-TZVPD-FINE level (BP_TZVPD_FINE_19 parameterization). The required quantum chemical calculations for all reactants, products and solvents were carried out as follows.

- BP86 geometry optimizations were performed with the def-TZVP basis set using the RI approximation for the evaluation of the Coulomb integrals, fine quadrature grids (5) and tight convergence criteria (SCF energy: $10^{-8}$ hartree, gradient: $10^{-5}$ hartree/bohr). Subsequent single-point energy calculations were done with the def2-TZVPD basis set.

- BP86 geometry optimizations within the COSMO model $(\varepsilon=\infty)$ were performed with the def-TZVP basis set using the RI approximation for the evaluation of the Coulomb 
integrals, fine quadrature grids (5) and tight convergence criteria (SCF energy: $10^{-8}$ hartree, gradient: $10^{-5}$ hartree/bohr). Subsequent single-point energy calculations within the COSMO model $(\varepsilon=\infty)$ were done with the def2-TZVPD basis set.

For the subsequent COSMO-RS calculations, the following solvent densities were used for the various temperatures:

- Acetonitrile

$$
\mathrm{T}=298 \mathrm{~K}: \rho=776.7 \mathrm{~g} / \mathrm{l} \quad \mathrm{T}=353 \mathrm{~K}: \rho=716.2 \mathrm{~g} / \mathrm{l}
$$

- Toluene

$$
\mathrm{T}=298 \mathrm{~K}: \rho=862.2 \mathrm{~g} / \mathrm{l} \quad \mathrm{T}=373 \mathrm{~K}: \rho=789.9 \mathrm{~g} / \mathrm{l}
$$

- tert-Butylbenzene

$$
\mathrm{T}=298 \mathrm{~K}: \rho=863.0 \mathrm{~g} / \mathrm{l} \quad \mathrm{T}=393 \mathrm{~K}: \rho=786.8 \mathrm{~g} / \mathrm{l}
$$




\section{Electronic Energies for the Dissociation and Exchange Reaction}

\subsection{Comparison of $\operatorname{CCSD}(\mathrm{T}) / \mathrm{CBS}$ and $\operatorname{CCSD}(\mathrm{T}) / \mathrm{TZVPP}$}

Table S1: Computed electronic energies $\left(\Delta E_{\text {gas }}\right)$ for the dissociation and exchange reaction. The mean absolute deviation (MAD) and the maximum absolute deviation (MAX) refer to the extrapolated $\operatorname{CCSD}(\mathrm{T})$ values. All values are given in $\mathrm{kJ} \mathrm{mol}^{-1}$.

\begin{tabular}{lrr}
\hline Compound & $\begin{array}{r}\text { CCSD(T) } \\
\text { CBS }\end{array}$ & $\begin{array}{r}\text { CCSD (T) } \\
\text { TZVPP }\end{array}$ \\
\hline NO-C bond dissociation & \\
$\mathbf{1 b}$ & 193.5 & 188.6 \\
$\mathbf{2 b}$ & 193.2 & 188.6 \\
$\mathbf{3 b}$ & 187.9 & 183.5 \\
$\mathbf{4 b}$ & 183.5 & 179.5 \\
$\mathbf{5 b}$ & 166.7 & 163.1 \\
$\mathbf{6 b}$ & 176.2 & 172.7 \\
$\mathbf{7 b}$ & 161.3 & 159.3 \\
$\mathbf{8 b}$ & 61.6 & 59.4 \\
MAD & 0.0 & 3.7 \\
MAX & 0.0 & 4.8 \\
& & \\
Exchange reaction & with nitroxide $\mathbf{2}$ \\
$\mathbf{1 b}$ & 0.3 & 0.0 \\
$\mathbf{3 b}$ & -5.3 & -5.1 \\
$\mathbf{4 b}$ & -9.7 & -9.1 \\
$\mathbf{5 b}$ & -26.4 & -25.5 \\
$\mathbf{6 b}$ & -16.9 & -15.9 \\
$\mathbf{7 b}$ & -31.9 & -29.3 \\
$\mathbf{8 b}$ & -131.6 & -129.2 \\
MAD & 0.0 & 1.1 \\
MAX & 0.0 & 2.6 \\
\hline
\end{tabular}




\subsection{Performance of Selected Density Functionals Using the QZVP Basis Set}

Table S2: Computed electronic energies $\left(\Delta E_{\text {gas }}\right)$ for the dissociation and exchange reaction. The mean absolute deviation (MAD) and the maximum absolute deviation (MAX) refer to the extrapolated $\operatorname{CCSD}(\mathrm{T})$ values. All values are given in $\mathrm{kJ} \mathrm{mol}^{-1}$.

\begin{tabular}{lrrrrr}
\hline Compound & $\begin{array}{r}\text { CCSD }(\mathrm{T}) \\
\text { CBS }\end{array}$ & $\begin{array}{r}\text { PBE } \\
\text { QZVP }\end{array}$ & $\begin{array}{r}\text { TPSS } \\
\text { QZVP }\end{array}$ & $\begin{array}{r}\text { B3LYP } \\
\text { QZVP }\end{array}$ & $\begin{array}{r}\text { M06-2X } \\
\text { QZVP }\end{array}$ \\
\hline NO-C bond dissociation & & & & \\
1b & 193.5 & 109.4 & 98.0 & 93.9 & 168.3 \\
$\mathbf{2 b}$ & 193.2 & 108.6 & 97.7 & 95.9 & 166.7 \\
$\mathbf{3 b}$ & 187.9 & 102.8 & 91.1 & 87.2 & 163.9 \\
$\mathbf{4 b}$ & 183.5 & 98.0 & 86.5 & 82.2 & 159.1 \\
$\mathbf{5 b}$ & 166.7 & 77.9 & 67.1 & 59.9 & 142.7 \\
$\mathbf{6 b}$ & 176.2 & 89.4 & 78.3 & 72.9 & 150.2 \\
$\mathbf{7 b}$ & 161.3 & 64.8 & 53.8 & 44.9 & 137.0 \\
$\mathbf{8 b}$ & 61.6 & -29.1 & -40.1 & -53.4 & 32.2 \\
MAD & 0.0 & 87.8 & 98.9 & 105.1 & 25.5 \\
MAX & 0.0 & 96.5 & 107.5 & 116.4 & 29.4 \\
& & & & & \\
Exchange reaction & with nitroxide $\mathbf{2}$ & & & \\
$\mathbf{1 b}$ & 0.3 & 0.8 & 0.4 & -2.1 & 1.6 \\
$\mathbf{3 b}$ & -5.3 & -5.7 & -6.6 & -8.7 & -2.8 \\
$\mathbf{4 b}$ & -9.7 & -10.6 & -11.1 & -13.7 & -7.7 \\
$\mathbf{5 b}$ & -26.4 & -30.7 & -30.6 & -36.1 & -24.0 \\
$\mathbf{6 b}$ & -16.9 & -19.2 & -19.4 & -23.0 & -16.5 \\
$\mathbf{7 b}$ & -31.9 & -43.8 & -43.8 & -51.0 & -29.7 \\
$\mathbf{8 b}$ & -131.6 & -137.6 & -137.7 & -149.3 & -134.6 \\
MAD & 0.0 & 3.8 & 3.9 & 8.9 & 2.0 \\
MAX & 0.0 & 11.9 & 12.0 & 19.1 & 3.0 \\
\hline
\end{tabular}




\section{Cartesian Atomic Coordinates of all Investigated Compounds}

The following optimized structures were obtained according to the method described in the Computational Details (B3LYP/TZVP). All coordinates are given in atomic length units (bohr).

\subsection{Nitroxide Radical Species}

\begin{tabular}{|c|c|c|}
\hline 1.80478092418996 & -3.71317790412972 & 0.00000000000000 \\
\hline 1.09238235140780 & -1.40792975277136 & 0.00000000000000 \\
\hline 0.29533223078442 & -0.35747221327637 & -2.51424816009124 \\
\hline 0.29533223078442 & -0.35747221327637 & 2.51424816009124 \\
\hline-0.00617462800553 & 2.52850181124593 & 2.34809843330065 \\
\hline-0.94935308175134 & 3.17088445566269 & 4.06988874705867 \\
\hline 1.87409778883201 & 3. 39246023064829 & 2.34783638598967 \\
\hline-0.00617462800553 & 2.52850181124593 & -2.34809843330065 \\
\hline 1.87409778883201 & 3.39246023064829 & -2.34783638598967 \\
\hline-0.94935308175134 & 3.17088445566269 & -4.06988874705867 \\
\hline-1.41977157817461 & 3.41424233823994 & 0.00000000000000 \\
\hline-1.55390953803498 & 5.47544441896052 & 0.00000000000000 \\
\hline-3.36246472503184 & 2.71007793447101 & 0.00000000000000 \\
\hline 2.38103386977597 & -0.99995714261369 & -4.41965452644622 \\
\hline 4.19186656976361 & -0.22573272406733 & -3.80741480197899 \\
\hline 2.58663031824475 & -3.03467539963613 & -4.63034220553353 \\
\hline 1.90792083682589 & -0.18125439812160 & -6.25388674886912 \\
\hline-2.18315070204368 & -1.63901907478197 & -3.34034736264101 \\
\hline-1.97861845107824 & -3.68578364241258 & -3.23275627631023 \\
\hline-3.78248596714301 & -1.08143983664490 & -2.16785357665014 \\
\hline-2.61760750138300 & -1.12584058338757 & -5.29141986851711 \\
\hline-2.18315070204368 & -1.63901907478197 & 3.34034736264101 \\
\hline-3.78248596714301 & -1.08143983664490 & 2.16785357665014 \\
\hline-1.97861845107824 & -3.68578364241258 & 3.23275627631023 \\
\hline-2.61760750138300 & -1.12584058338757 & 5.29141986851711 \\
\hline 2.38103386977597 & -0.99995714261369 & 4.41965452644622 \\
\hline 2.58663031824475 & -3.03467539963613 & 4.63034220553353 \\
\hline 4.19186656976361 & -0.22573272406733 & 3.80741480197899 \\
\hline 1.90792083682589 & -0.18125439812160 & 6.25388674886912 \\
\hline
\end{tabular}

TMIO, 2
0.00000000000000
0.00000000000000
0.00000000000000
0.00000000000000
0.00000000000000
0.00000000000000
0.00000000000000
0.00000000000000
0.00000000000000
0.00000000000000
0.00000000000000
0.00000000000000
2.38318276385682
4.08804137275726
2. 41722049176522
$-1.31609045509235$
1.31609045509235
2. 33418611919199
$-2.64503783252673$
$-4.69292780433144$
$-1.31706815142370$
1. 31706815142370
2.64503783252673
4. 69292780433144
2. 38189872746978
$-2.38189872746978$
0.00000000000000
3.91687894454126
2.85983796559649
4. 35847793996955

$\begin{array}{rc}5.84728460225975 & \mathrm{c} \\ 5.84728460225975 & \mathrm{c} \\ 7.62259280718823 & \mathrm{~h} \\ 3.57792528899598 & \mathrm{c} \\ 3.59077006552319 & \mathrm{~h} \\ 1.31346025905588 & \mathrm{c} \\ 1.31346025905588 & \mathrm{c} \\ 3.57792528899598 & \mathrm{c} \\ 3.59077006552319 & \mathrm{~h} \\ -1.33512403669772 & \mathrm{c} \\ -1.33512403669772 & \mathrm{c} \\ -2.83649589595274 & \mathrm{n} \\ -1.95430162825604 & \mathrm{c} \\ -1.48124724471382 & \mathrm{~h} \\ -3.96699496854563 & \mathrm{~h}\end{array}$




\begin{tabular}{rrrr}
2.39513953679026 & 5.68326630603584 & -0.88945904445797 & $\mathrm{~h}$ \\
-2.38318276385682 & 3.91687894454126 & -1.95430162825604 & $\mathrm{c}$ \\
-2.41722049176522 & 4.35847793996955 & -3.96699496854563 & $\mathrm{~h}$ \\
-2.39513953679026 & 5.68326630603584 & -0.88945904445797 & $\mathrm{~h}$ \\
-4.08804137275726 & 2.85983796559649 & -1.48124724471382 & $\mathrm{~h}$ \\
-2.38318276385682 & -3.91687894454126 & -1.95430162825604 & $\mathrm{c}$ \\
-2.39513953679026 & -5.68326630603584 & -0.88945904445797 & $\mathrm{~h}$ \\
-2.41722049176522 & -4.35847793996955 & -3.96699496854563 & $\mathrm{~h}$ \\
-4.08804137275726 & -2.85983796559649 & -1.48124724471382 & $\mathrm{~h}$ \\
2.38318276385682 & -3.91687894454126 & -1.95430162825604 & $\mathrm{c}$ \\
2.41722049176522 & -4.35847793996955 & -3.96699496854563 & $\mathrm{~h}$ \\
4.08804137275726 & -2.85983796559649 & -1.48124724471382 & $\mathrm{~h}$ \\
2.39513953679026 & -5.68326630603584 & -0.88945904445797 & $\mathrm{~h}$ \\
0.00000000000000 & 0.00000000000000 & -5.22931053280426 & $\mathrm{o}$ \\
0.00000000000000 & -2.33418611919199 & 7.62259280718823 & $\mathrm{~h}$ \\
\hline--------------------------------------------------
\end{tabular}

$\begin{array}{rrrr}1.15879473017875 & -3.71472621684828 & -0.46795638054551 & \mathrm{o} \\ 0.46878870817863 & -1.40367705855904 & -0.39779745009425 & \mathrm{n} \\ -0.59748285545017 & -0.35456911016212 & -2.80945020226780 & \mathrm{c} \\ 0.00816438806127 & -0.33194060868679 & 2.18657752103335 & \mathrm{c} \\ -0.35321934611721 & 2.55009376225147 & 2.05405681987466 & \mathrm{c} \\ -1.11845137517952 & 3.17241839629845 & 3.86898267039057 & \mathrm{~h} \\ 1.48671030611916 & 3.46446130991176 & 1.84508784220235 & \mathrm{~h} \\ -0.88362733767734 & 2.53024754985378 & -2.61235900417692 & \mathrm{c} \\ 0.98302038334952 & 3.39902798470411 & -2.81822890321154 & \mathrm{~h} \\ -2.01152502709305 & 3.16963379053975 & -4.22034945175150 & \mathrm{~h} \\ -2.03401986054798 & 3.40952936110778 & -0.12315432366947 & \mathrm{c} \\ -2.19001882925815 & 5.46919715740212 & -0.11265656449651 & \mathrm{~h} \\ -3.95636400137087 & 2.68432879649258 & 0.09505113687596 & \mathrm{~h} \\ 1.26434536424362 & -0.99721303260139 & -4.93404640448391 & \mathrm{c} \\ 3.12891483066119 & -0.21194286376791 & -4.53340719537760 & \mathrm{~h} \\ 1.45342399934747 & -3.03224534249517 & -5.15793922351719 & \mathrm{~h} \\ -3.15080357617906 & -1.64042072286163 & -3.35229968545429 & \mathrm{c} \\ -2.92912823619949 & -3.68681382803967 & -3.27921883502804 & \mathrm{~h} \\ -4.60499971824144 & -1.09475204654944 & -1.99889182406782 & \mathrm{~h} \\ -2.34871708213105 & -1.61150009247747 & 3.32077523745285 & \mathrm{c} \\ -4.08419209188644 & -1.03496011620474 & 2.37198077335881 & \mathrm{~h} \\ -2.17127046710035 & -3.65787121418601 & 3.17042044908438 & \mathrm{~h} \\ 2.30235013970678 & -1.00505264514466 & 3.86873836831651 & \mathrm{c} \\ 1.97036024377856 & -0.10277091276939 & 5.70059407282807 & \mathrm{~h} \\ 2.27528366431376 & -3.03973928193562 & 4.19410837329965 & \mathrm{~h} \\ 4.90656441611684 & -0.25901058750382 & 2.87172435633344 & \mathrm{c} \\ 5.09078475198883 & 1.77344847310333 & 2.56543960767579 & \mathrm{~h} \\ 6.35834001334247 & -0.80152958530302 & 4.23459195148744 & \mathrm{~h} \\ 5.32275924214958 & -1.22102429799071 & 1.09836962243626 & \mathrm{~h} \\ 0.58529028709135 & -0.18913961352346 & -6.70724765866928 & \mathrm{~h} \\ -3.80876731780617 & -1.11879459789265 & -5.23740586984477 & \mathrm{~h} \\ -2.52130834638959 & -1.11269280616220 & 5.31591017400626 & \mathrm{~h} \\ & & & \end{array}$

$\begin{array}{rrrr}2.24814808157220 & -3.21531129242088 & -0.83730083689562 & \circ \\ 0.82436264471095 & -1.26874505966911 & -0.90351822103690 & \mathrm{n} \\ -0.24843512934709 & -0.62314271574627 & -3.45221419030750 & \mathrm{c}\end{array}$




\begin{abstract}
$-0.22062504500868$
$-1.61496994209559$

$-2.76777262589832$

$-0.24239071092806$

$-1.57499667689456$

$-0.14059105640045$

$-2.66616215406289$

$-3.23866491451525$

$-4.15095853752927$

$-4.76408589685828$

1. 96343222339455

3. 35441331140652

$-2.08462317472782$

$-3.79508979037843$

$-2.04637619233435$

$-3.67573302183710$

2. 06902772476959

1. 30201979252603

2.78497889875498

4. 25265704282394

3. 71648480903401

5.82120598712260

4. 95162205932485

2.93625737308297

0.65834486532720

$-0.18110453725031$

2.89979968147562

1. 25952561512808

$-1.15826540160767$

$-2.64426051740624$

$-1.07240827341433$

$-2.75476651195947$
\end{abstract}

$-0.41485903786795$

2. 12313883797801

2. 40585222563053

3. 66608670845399

1. 95814910092116

3. 44941060363318

2. 16950696408582

2. 31360737364021

4. 16569461031901

0.92047515680757

$-0.51669322142952$

0.88614582440604

$-2.73003991430567$

$-2.76023278331528$

$-2.49032559941177$

$-2.66893336324374$

$-0.19688944935100$

0.75776735272812

$-2.12055419135263$

1. 41164806110348

3. 39138873538742

1. 37487118034744

0.69483649830777

0.68542160168706

2.71936870242175

$-0.37988626426535$

$-2.34216044139806$

0.01526142025431

$-4.56193572392252$

$-2.44967686255360$

$-4.30430086599574$

$-2.06494417186379$
$1.60133859687456 \quad \mathrm{c}$

$1.32445541127616 \quad \mathrm{c}$

$3.01170102058623 \mathrm{~h}$

$1.28434217813276 \mathrm{~h}$

-3.36974241253675 c

$-3.38781936593532 \mathrm{~h}$

$-5.11076525829298 \mathrm{~h}$

-1.04905671823411 c

$-1.11571735706714 \mathrm{~h}$

$-1.01683680037058 \mathrm{~h}$

$-5.32139378923055 \mathrm{c}$

$-4.72862166541787 \mathrm{~h}$

-4.26259376636315 c

$-3.11440392886329 \mathrm{~h}$

2.52029839455019 c

1.27239964359822 h

3.46365825469871 c

$6.09293868154564 \quad \mathrm{c}$

$3.67121688811260 \mathrm{~h}$

$2.44250735344332 \mathrm{c}$

$2.19578420506811 \mathrm{~h}$

$3.78390844868641 \mathrm{~h}$

$0.64525999004358 \mathrm{~h}$

$7.35103049324766 \mathrm{~h}$

$6.04612157353880 \mathrm{~h}$

$6.96003595058962 \mathrm{~h}$

$-5.45768748619323 \mathrm{~h}$

$-7.18687701702190 \mathrm{~h}$

$-4.09905131316770 \mathrm{~h}$

$-6.22899952763687 \mathrm{~h}$

$2.56918558057828 \mathrm{~h}$

$4.40641699000074 \mathrm{~h}$

$\begin{array}{rrrr}-0.95251976038121 & -3.65267289347970 & -0.70636144283560 & \mathrm{o} \\ -0.23594035384383 & -1.37751324803129 & -1.06479889303173 & \mathrm{~h} \\ 0.26576149067577 & -0.72434567754944 & -3.79291901727528 & \mathrm{c} \\ -0.77201708168291 & 0.46747896841078 & 1.04214994222927 & \mathrm{c} \\ 0.37088311754416 & 3.07435944923128 & 0.39011287435018 & \mathrm{c} \\ -0.50255308276360 & 4.47381502270192 & 1.62555939001433 & \mathrm{~h} \\ 2.38781996205677 & 3.08050910851200 & 0.82876662222456 & \mathrm{~h} \\ 1.39677591090203 & 1.93354602711343 & -4.03601910672556 & \mathrm{c} \\ 3.39928485661339 & 1.86821955031749 & -3.51892216016948 & \mathrm{~h} \\ 1.32002897787925 & 2.48098866228042 & -6.02609471286726 & \mathrm{~h} \\ 0.07734145871918 & 3.86147950498857 & -2.36435114184902 & \mathrm{c} \\ 0.92020770013954 & 5.72819546570765 & -2.63348677573651 & \mathrm{~h} \\ -1.91342958147798 & 4.04855184459503 & -2.88318591165856 & \mathrm{~h} \\ 2.14607446603410 & -2.67758366288527 & -4.81605329688434 & \mathrm{c} \\ -2.23618193475982 & -0.93517012695592 & -5.26290290763403 & \mathrm{c} \\ -3.66478831765908 & 0.71287070559004 & 1.26052575091529 & \mathrm{c} \\ 0.43551482364153 & -0.57501247953034 & 3.60791130466418 & \mathrm{c} \\ 0.59551858786822 & 1.56975669750415 & 5.58241739414409 & \mathrm{c} \\ -1.13263431012340 & -2.70904275369143 & 4.82902033047181 & \mathrm{c} \\ 3.12475997908324 & -1.58096914580890 & 3.14029145095233 & \mathrm{c} \\ 4.33523198243640 & -0.18885476158330 & 2.21425362278810 & \mathrm{~h} \\ 4.00694027069366 & -2.05222270666870 & 4.94635334739259 & \mathrm{~h}\end{array}$




\begin{abstract}
3.09771279086353
1. 20980537783748

1. 94966887914354

$-1.23283719422819$

$-1.39702582593414$

$-0.14208128483221$

$-2.98521247192106$

2.59120062322770

1. 36041019171681

3. 89967203634500

$-1.87512273836195$

$-3.58600118154109$

$-3.09224391146725$

$-4.44053913569973$

$-4.18265009499641$

$-4.54683522174750$
\end{abstract}

$-3.28403194298416$

0.77123932028051

3.03541237132204

2. 46904025728201

$-4.29063630324255$

$-3.38228785822712$

$-2.04348680540277$

$-2.23530541956625$

$-4.57520837885631$

$-2.64184272715084$

$-0.74134856326609$

0.52212385989335

$-2.77583558088903$

1.73156579657791

1.75670561365984

$-1.14248719019905$
$1.98515903895381 \mathrm{~h}$

$7.38312901398968 \mathrm{~h}$

$5.07162143886520 \mathrm{~h}$

$5.91044856875312 \mathrm{~h}$

$3.54810612241237 \mathrm{~h}$

$6.51196941373727 \mathrm{~h}$

$5.44502839098866 \mathrm{~h}$

$-6.78209024986668 \mathrm{~h}$

$-4.72166496932249 \mathrm{~h}$

$-3.73065812124402 \mathrm{~h}$

$-7.28540094888633 \mathrm{~h}$

$-4.71420922485140 \mathrm{~h}$

$-4.92076779318193 \mathrm{~h}$

$-0.35426878691879 \mathrm{~h}$

$2.96075765081448 \mathrm{~h}$

1.33057379227772 h

\begin{tabular}{|c|c|c|c|}
\hline 1.42076824181192 & -3.51112115159225 & 0.00000000000000 & o \\
\hline 0.70590635191528 & -1.20610289697762 & 0.00000000000000 & $\mathrm{n}$ \\
\hline-0.05615882958816 & -0.14930307108315 & -2.52903558581633 & c \\
\hline-0.05615882958816 & -0.14930307108315 & 2.52903558581633 & c \\
\hline-0.36885007582747 & 2.73482926888001 & 2.35235565254869 & c \\
\hline-1.31638187177834 & 3. 38850525404921 & 4.06475316210102 & $\mathrm{~h}$ \\
\hline 1.49647476899446 & 3.62168203336236 & 2.34985338538951 & $\mathrm{~h}$ \\
\hline-0.36885007582747 & 2.73482926888001 & -2.35235565254869 & c \\
\hline 1.49647476899446 & 3.62168203336236 & -2.34985338538951 & $\mathrm{~h}$ \\
\hline-1.31638187177834 & 3.38850525404921 & -4.06475316210102 & $\mathrm{~h}$ \\
\hline-1.78294010888559 & 3.61045733691544 & 0.00000000000000 & c \\
\hline-1.92035266214606 & 5.67184289939872 & 0.00000000000000 & $\mathrm{~h}$ \\
\hline-3.72494116895991 & 2.90587011546925 & 0.00000000000000 & $\mathrm{~h}$ \\
\hline 2.02142605250279 & -0.83322324440834 & 4.46950215654864 & c \\
\hline 4.74054247078150 & -0.14939158136859 & 3.78161016918886 & c \\
\hline 1.92349095690052 & -2.86094471004393 & 4.82980852483563 & $\mathrm{~h}$ \\
\hline 1.51564368464182 & 0.11127545268811 & 6.23537806456664 & $\mathrm{~h}$ \\
\hline 5.39081958081786 & -1.22897967267667 & 2.15565163937527 & $\mathrm{~h}$ \\
\hline 5.98785822814463 & -0.59009208691109 & 5.36614017634750 & $\mathrm{~h}$ \\
\hline 4.97814272890827 & 1.85681515034134 & 3.36158340863894 & $\mathrm{~h}$ \\
\hline 2.02142605250279 & -0.83322324440834 & -4.46950215654864 & c \\
\hline 4.74054247078150 & -0.14939158136859 & -3.78161016918886 & c \\
\hline 1.92349095690052 & -2.86094471004393 & -4.82980852483563 & $\mathrm{~h}$ \\
\hline 1.51564368464182 & 0.11127545268811 & -6.23537806456664 & $\mathrm{~h}$ \\
\hline 5.98785822814463 & -0.59009208691109 & -5.36614017634750 & $\mathrm{~h}$ \\
\hline 4.97814272890827 & 1.85681515034134 & -3.36158340863894 & $\mathrm{~h}$ \\
\hline 5.39081958081786 & -1.22897967267667 & -2.15565163937527 & $\mathrm{~h}$ \\
\hline-2.56922355293632 & -1.46714536943121 & 3.30379429508660 & c \\
\hline-3.48526207455502 & -1.02496065664526 & 6.01102030915159 & c \\
\hline-2.29401906495375 & -3.48933558005697 & 2.99834153523747 & $\mathrm{~h}$ \\
\hline-4.06102090975159 & -0.87705379113923 & 2.00739853916596 & $\mathrm{~h}$ \\
\hline-3.74805639304215 & 0.97721273988146 & 6.43969863643496 & $\mathrm{~h}$ \\
\hline-2.19258842070750 & -1.80803192033929 & 7.41144792866921 & $\mathrm{~h}$ \\
\hline-5.31205760541944 & -1.94733136670551 & 6.28052772045701 & $\mathrm{~h}$ \\
\hline-2.56922355293632 & -1.46714536943121 & -3.30379429508660 & c \\
\hline-3.48526207455502 & -1.02496065664526 & -6.01102030915159 & c \\
\hline-4.06102090975159 & -0.87705379113923 & -2.00739853916596 & $\mathrm{~h}$ \\
\hline-2.29401906495375 & -3.48933558005697 & -2.99834153523747 & $\mathrm{~h}$ \\
\hline
\end{tabular}


$-3.74805639304215$

$-2.19258842070750$

$-5.31205760541944$
0.97721273988146

$-1.80803192033929$

$-1.94733136670551$
$-6.43969863643496 \mathrm{~h}$

$-7.41144792866921 \mathrm{~h}$

$-6.28052772045701 \mathrm{~h}$

\begin{tabular}{|c|c|c|}
\hline 1.83096127862168 & -3.08824292364025 & -0.21501436394375 \\
\hline 0.89788882023844 & -0.86182152239860 & -0.11544153486011 \\
\hline-0.19716178799973 & 0.15037293613786 & -2.58295153738569 \\
\hline 0.48412092031964 & 0.23845297787075 & 2.52947493037748 \\
\hline 0.14589051106683 & 3.11319723913672 & 2.28376218990544 \\
\hline-0.47622949043765 & 3.87615525807487 & 4.09055939243630 \\
\hline 1.98038854847842 & 3.97369285826695 & 1.89821184532376 \\
\hline-0.51037747932058 & 3.03101337919140 & -2.35127643379810 \\
\hline 1.34289684228052 & 3.92080554209876 & -2.53739051825211 \\
\hline-1.63247295508099 & 3.71592565581477 & -3.93256211991459 \\
\hline-1.63461334970702 & 3.88999823303496 & 0.15792419451099 \\
\hline-1.84676042778476 & 5.94546093058054 & 0.14913677463908 \\
\hline-3.52747039436660 & 3.11065762630142 & 0.43898532559612 \\
\hline 2.91658066391699 & -0.40200242555559 & 4.09963185910075 \\
\hline 3.11303152990146 & -2.44759971097278 & 3.99601485488330 \\
\hline 2.76546091001478 & 0.33435598598776 & 6.90347147462819 \\
\hline 5.36049310100978 & 0.72098310262232 & 3.01054795290293 \\
\hline 5.50642 & 2.75214016090351 & 3.35 \\
\hline 6.98005639398143 & -0.16185641702562 & 3.93686310206294 \\
\hline 5.54754942834012 & 0.39359562411857 & 0.99370120339853 \\
\hline 4.58741464762883 & -0.05674141598672 & 7.79202002583116 \\
\hline 2.38669797304456 & 2.34738534770829 & 7.16571341049893 \\
\hline 1.34801131677610 & -0.72661547440433 & 7.94734025738297 \\
\hline 1.74186303967332 & -0.51279294020474 & -4.73265364685739 \\
\hline 1.78410287393532 & -2.56601426009974 & -4.84387193603789 \\
\hline 0.95647048664061 & 0.48853627393998 & -7.34248665326967 \\
\hline 0.84061742135146 & 2.54932102357482 & -7.39128490610101 \\
\hline 2.37836023732161 & -0.07054086798899 & -8.72989411801441 \\
\hline-0.84324416175404 & -0.26611793348435 & -7.98872354213920 \\
\hline 4.47184733216664 & 0.30366731633533 & -4.19927346788383 \\
\hline 4.66626281891267 & 2.33837498093135 & -3.90909963103493 \\
\hline 5.24350106301498 & -0.67483092232059 & -2.56789919086580 \\
\hline 5.64900002571894 & -0.18682119637599 & -5.82285715190038 \\
\hline-1.98284494342578 & -1.04648452080292 & 3.65914818515532 \\
\hline-3.32783216284245 & -1.07093332420700 & 2.10348726457973 \\
\hline-1.62936422025360 & -3.81946097084937 & 4.43894783485475 \\
\hline-0.53104492127075 & -4.00707381580324 & 6.17476546663618 \\
\hline-3.47784704323376 & -4.66897590665162 & 4.79530271664469 \\
\hline-0.69096914019554 & -4.91044606401566 & 2.96940599076943 \\
\hline-3.35795385039428 & 0.42034858681726 & 5.76383856826554 \\
\hline-2.21826549629085 & 0.70673842039701 & 7.45202561179134 \\
\hline-4.02594526220594 & 2.26425285302595 & 5.13165443043378 \\
\hline-5.02484123642847 & -0.65968560956816 & 6.32959037389153 \\
\hline-2.84919717968402 & -1.21170703881365 & -3.01231326796947 \\
\hline-3.68167722642776 & -1.35466965138229 & -1.13838309795854 \\
\hline-2.64793555118531 & -3.93939253787132 & -3.98322238084182 \\
\hline-1.28097722546342 & -5.03316402603123 & -2.90277689105971 \\
\hline-4.48876929507437 & -4.85971418345735 & -3.81102374548863 \\
\hline-2.11446656351324 & -4.01860411590895 & -5.97506748304810 \\
\hline-4.82095612144488 & 0.24931803730070 & -4.57507616365419 \\
\hline-6.54615850551761 & -0.88147649588126 & -4.67374177383402 \\
\hline
\end{tabular}


$-5.33364533878133$

$-4.22687122923327$
2.05219555657834

0.61684036495245
$-3.71961187065759 \mathrm{~h}$

$-6.51142185474826 \mathrm{~h}$

\begin{tabular}{|c|c|c|}
\hline 0.00000000000000 & 0.00000000000000 & -3.31831220696966 \\
\hline-0.00000000000000 & 0.00000000000000 & -0.90661617143846 \\
\hline 0.22134119207400 & 2.64222184547288 & 0.34288389296722 \\
\hline-0.22134119207400 & -2.64222184547288 & 0.34288389296722 \\
\hline-1.09904448490385 & -2.22824729223297 & 3.09610662891573 \\
\hline-3.13712150486464 & -2.00264002619167 & 3.06371207035362 \\
\hline-0.77565780014554 & -3.96689410028371 & 4.15039331097979 \\
\hline 1.09904448490385 & 2.22824729223297 & 3.09610662891573 \\
\hline 3.13712150486464 & 2.00264002619167 & 3.06371207035362 \\
\hline 0.77565780014554 & 3.96689410028371 & 4.15039331097979 \\
\hline-0.00000000000000 & 0.00000000000000 & 4.61000220542998 \\
\hline 1.49063529332004 & -0.66963603596949 & 5.86244253337698 \\
\hline-1.49063529332004 & 0.66963603596949 & 5.86244253337698 \\
\hline 2.47717580360528 & 4.22693880945681 & -1.10120483755543 \\
\hline 3.63057508710971 & 6.30792152334274 & 0.62194799294712 \\
\hline 4.74479172552094 & 2.54482953049355 & -1.81353182292414 \\
\hline 1.72313798875854 & 5.50483046138168 & -3.61753370039116 \\
\hline-2.48403891514678 & 4.13218666497936 & 0.31309332563786 \\
\hline-2.24615832414177 & 6.94790492594953 & 1.07855425442533 \\
\hline-3.86834125124731 & 4.09066714745065 & -2.25453660003940 \\
\hline-4.35887028541220 & 3.08107781326070 & 2.29340774813173 \\
\hline-2.47717580360528 & -4.22693880945681 & -1.10120483755543 \\
\hline-3.63057508710971 & -6.30792152334274 & 0.62194799294712 \\
\hline-4.74479172552094 & -2.54482953049355 & -1.81353182292414 \\
\hline-1.72313798875854 & -5.50483046138168 & -3.61753370039116 \\
\hline 2.48403891514678 & -4.13218666497936 & 0.31309332563786 \\
\hline 2.24615832414177 & -6.94790492594953 & 1.07855425442533 \\
\hline 3.86834125124731 & -4.09066714745065 & -2.25453660003940 \\
\hline 4.35887028541220 & -3.08107781326070 & 2.29340774813173 \\
\hline 3.73231535395533 & -3.44136059258419 & 4.22049141400233 \\
\hline 6.16254640326313 & -4.05425493728835 & 2.06118118391605 \\
\hline 4.73297397993626 & -1.08070346453955 & 2.09400842898680 \\
\hline-3.43997445938073 & -6.25820353639188 & -4.48465670704631 \\
\hline-0.89932137789580 & -4.15227221388125 & -4.92366239909450 \\
\hline-0.44061206748261 & -7.09299934077359 & -3.39002226656952 \\
\hline-4.73297397993626 & 1.08070346453955 & 2.09400842898680 \\
\hline-3.73231535395533 & 3.44136059258419 & 4.22049141400233 \\
\hline-6.16254640326313 & 4.05425493728835 & 2.06118118391605 \\
\hline 0.44061206748261 & 7.09299934077359 & -3.39002226656952 \\
\hline 3.43997445938073 & 6.25820353639188 & -4.48465670704631 \\
\hline 0.89932137789580 & 4.15227221388125 & -4.92366239909450 \\
\hline 4.15511930500719 & -7.72457315006641 & 1.19836378120471 \\
\hline 1.38800302784726 & -7.19691747682976 & 2.93575538760164 \\
\hline 1.22132405116966 & -8.09687194096661 & -0.27595436993551 \\
\hline-4.28970088452172 & -1.30610561932269 & -3.38178648773000 \\
\hline-6.29065577928332 & -3.77078731055917 & -2.41906341441053 \\
\hline-5.46167122783701 & -1.42083446104219 & -0.25175570496663 \\
\hline-1.22132405116966 & 8.09687194096661 & -0.27595436993551 \\
\hline-4.15511930500719 & 7.72457315006641 & 1.19836378120471 \\
\hline-1.38800302784726 & 7.19691747682976 & 2.93575538760164 \\
\hline 6.29065577928332 & 3.77078731055917 & -2.41906341441053 \\
\hline 5.46167122783701 & 1.42083446104219 & -0.25175570496663 \\
\hline
\end{tabular}




\begin{tabular}{|c|c|c|}
\hline 4.28970088452172 & 1.30610561932269 & -3.38178648773000 \\
\hline 4.71126484657813 & 5.51835754064907 & 2.18753791578437 \\
\hline 4.95739405835274 & 7.40077662972363 & -0.51993925742272 \\
\hline 2.27377612458561 & 7.63231599913214 & 1.39666453553051 \\
\hline 4.19025552926735 & -2.19415707458800 & -2.94523393926793 \\
\hline 5.71188783310935 & -4.99022710933010 & -2.00816621258948 \\
\hline 2.88113137808379 & -5.12288607745450 & -3.72203359832932 \\
\hline-2.27377612458561 & -7.63231599913214 & 1.39666453553051 \\
\hline-4.71126484657813 & -5.51835754064907 & 2.18753791578437 \\
\hline-4.95739405835274 & -7.40077662972363 & -0.51993925742272 \\
\hline-2.88113137808379 & 5.12288607745450 & -3.72203359832932 \\
\hline-4.19025552926735 & 2.19415707458800 & -2.94523393926793 \\
\hline-5.71188783310935 & 4.99022710933010 & -2.00816621258948 \\
\hline
\end{tabular}

\subsection{Carbon Radical Species}

Ben, a

\begin{tabular}{|c|c|c|}
\hline 0.00000000000000 & 0.00000000000000 & -1.73728427655444 \\
\hline-2.29420544756181 & 0.00000000000000 & -0.33581455909762 \\
\hline-2.28199610913849 & 0.00000000000000 & 2.27235260896223 \\
\hline 0.00000000000000 & 0.00000000000000 & 3.60158387375427 \\
\hline 2.28199610913849 & 0.00000000000000 & 2.27235260896223 \\
\hline 2.29420544756181 & 0.00000000000000 & -0.33581455909762 \\
\hline-4.07097222439644 & 0.00000000000000 & -1.35438956412906 \\
\hline-4.05503961102077 & 0.00000000000000 & 3.29607141417840 \\
\hline 0.00000000000000 & 0.00000000000000 & 5.64734582431687 \\
\hline 4.05503961102077 & 0.00000000000000 & 3.29607141417840 \\
\hline 4.07097222439644 & 0.00000000000000 & -1.35438956412906 \\
\hline 0.00000000000000 & 0.00000000000000 & -4.38535371760508 \\
\hline-1.74997465826408 & 0.00000000000000 & -5.44136575186972 \\
\hline 1.74997465826408 & 0.00000000000000 & -5.44136575186972 \\
\hline
\end{tabular}

PhEt, b

$\begin{array}{rrrr}-2.85903557100589 & -3.40210625150282 & 0.00000000000000 & \mathrm{c} \\ -4.65185378503357 & -4.39103172266365 & 0.00000000000000 & \mathrm{~h} \\ -2.82036265960913 & -0.79453510521073 & 0.00000000000000 & \mathrm{c} \\ -4.57999308770078 & 0.25457451243697 & 0.00000000000000 & \mathrm{~h} \\ -0.50724758608758 & 0.57165522190503 & 0.00000000000000 & \mathrm{c} \\ 1.75880279906517 & -0.86831716788967 & 0.00000000000000 & \mathrm{c} \\ 3.56339864151316 & 0.09474833329075 & 0.00000000000000 & \mathrm{~h} \\ 1.69715523990858 & -3.48054860006399 & 0.00000000000000 & \mathrm{c} \\ 3.45393490365013 & -4.53263005201771 & 0.00000000000000 & \mathrm{~h} \\ -0.60168167473745 & -4.77298956381963 & 0.00000000000000 & \mathrm{c} \\ -0.63585512893855 & -6.81851036848023 & 0.00000000000000 & \mathrm{~h} \\ -0.51781707742051 & 3.23688560231896 & 0.00000000000000 & \mathrm{c} \\ -2.34333925982426 & 4.16624543049206 & 0.00000000000000 & \mathrm{~h} \\ 1.78836617378425 & 4.85784591570200 & 0.00000000000000 & \mathrm{c} \\ 2.98200923032734 & 4.51114532522774 & -1.65863495730171 & \mathrm{~h} \\ 1.29150961178172 & 6.85642316504720 & 0.00000000000000 & \mathrm{~h} \\ 2.98200923032734 & 4.51114532522774 & 1.65863495730171 & \mathrm{~h} \\ -------------------------------------------------\end{array}$


Cum, c
0.00000000000000
2.27202716673854
-4.20210043891713 c
0.00000000000000
0.00000000000000
0.00000000000000
$-2.27202716673854$
0.00000000000000
$-2.28288211103641$
-5.53859390008892 c
-4.20210043891713 c
$-1.58932170125722 \quad \mathrm{c}$
0.00000000000000
0.00000000000000
0.00000000000000
2. 28288211103641
0.00000000000000
0.00000000000000
0.00000000000000
$-2.43765853654853$
0.00000000000000
2.43765853654853
0.00000000000000
$-4.05092670635026$
0.00000000000000
$-4.07683811846066$
0.00000000000000
4.07683811846066
0.00000000000000
4.05092670635026
0.00000000000000
0.00000000000000
$-1.65745590442908$
3. 60220748381414
-0.17470873639179 c
$-1.58932170125722 \quad \mathrm{c}$
$2.51668265399758 \quad \mathrm{c}$
3.96277992536302 c
$3.96277992536302 \mathrm{c}$
$-5.21651762100332 \mathrm{~h}$
$-0.61028471959587 \mathrm{~h}$
$-0.61028471959587 \mathrm{~h}$
$-5.21651762100332 \mathrm{~h}$
$-7.58441884756817 \mathrm{~h}$
$3.52602851789287 \mathrm{~h}$
2.09977054899913
$5.99390693465044 \mathrm{~h}$
1.65745590442908
3. 60220748381414
0.00000000000000
$-2.09977054899913$
$-1.65745590442908$
1.65745590442908
$-3.60220748381414$
$-3.60220748381414$
$3.52602851789287 \mathrm{~h}$
$5.99390693465044 \mathrm{~h}$
$3.52602851789287 \mathrm{~h}$
$3.52602851789287 \mathrm{~h}$

\subsection{Alkoxyamines}

$1 a$
8.04032071185301
9.35055322820600
3. 95333905447555
4. 11000721574811
8.04032071185301
3. 95333905447555
5.43239796180887
3. 63876431590978
4.09693019327254
5.43239796180887
3. 47605458511107
3. 63876431590978
1. 27853436542944
3. 09481485761324
0.81756800300349
$-1.85109470460735$
0.41959331731171
$-0.13412703588519$
$-2.47682217769744$
$-5.30567063103820$
$-1.41717159813893$
$-2.10816473440756$
$-6.05837329062518$
$-5.30567063103820$
$-2.47682217769744$
$-0.84571200350982$
$-2.10065759418619$
$-0.84571200350982$
$-2.10065759418619$
$-8.09796834648641$
$-5.17401292355263$
$-6.40374549232010$
$-6.40374549232010$
$-5.76126456540935$
$-5.76126456540935$
$-2.83554983732245$
$-2.83554983732245$
$-3.10636147355846$
$-3.59020702093525$
$-2.10816473440756$
$-1.41717159813893$
$-3.75402681127118$
0.51501831075757
$-3.75402681127118$
0.51501831075757
$-3.92204148142181$
$-5.45745451836399$
$-0.35596250561349$
$-0.35596250561349$
$-3.16298631366826$
$-3.16298631366826$
$-0.25468704585864$
$-0.25468704585864$
2. 26638018450278
$-3.10636147355846$
2. 26638018450278 


\begin{abstract}
$-0.10582942787039$
$-0.10582942787039$

$-1.10877192060491$

$-1.10877192060491$

$-1.33413492168935$

$-1.33413492168935$

1. 14878787539385

1. 14878787539385

0.42382199197618

4.41806057216620

9.04905112075415

11.38202309786887

4.41806057216620

9.04905112075415

0.42382199197618
\end{abstract}

0.94064461681787

0.94064461681787

$-4.26374594961889$

$-4.26374594961889$

$-5.40246623717112$

$-5.40246623717112$

$-3.33589185495763$

$-3.33589185495763$

3. 95596326137448

3. 52440959030425

4.08196946636642

4.36058858121634

3.52440959030425

4.08196946636642

3. 95596326137448
$4.85875370761477 \mathrm{~h}$

$-4.85875370761477 \mathrm{~h}$

$5.04250565200788 \mathrm{~h}$

$-5.04250565200788 \mathrm{~h}$

$1.93266510301733 \mathrm{~h}$

$-1.93266510301733 \mathrm{~h}$

$2.76776964485718 \mathrm{~h}$

$-2.76776964485718 \mathrm{~h}$

$-1.66938409231038 \mathrm{~h}$

$4.04314063117856 \mathrm{~h}$

$4.04962926544947 \mathrm{~h}$

$0.00000000000000 \mathrm{~h}$

$-4.04314063117856 \mathrm{~h}$

$-4.04962926544947 \mathrm{~h}$

$1.66938409231038 \mathrm{~h}$

$1 b$

\begin{tabular}{|c|c|c|}
\hline-0.84119929508651 & -0.69671568665831 & 1.15626676990631 \\
\hline 1.75605565740848 & -0.48067514115657 & 0.33188947350548 \\
\hline 2.74908114235071 & 2.06393657526138 & 0.42479861556944 \\
\hline-5.15349544816878 & -3.77954810918378 & 2.57047715362872 \\
\hline-7.11636673178548 & -3.93741981194102 & 3.19731773467577 \\
\hline-4.45566595501884 & -5.71404282528102 & 2.37282019790473 \\
\hline 2.59643284360143 & 3.44460873263144 & -2.08462446813121 \\
\hline 5.49122610021061 & 1.83092074299603 & 1.29859634711998 \\
\hline 0.69815619991802 & -4.35955233880561 & 3.94193768066843 \\
\hline-1.16574221072786 & -4.42512748632545 & -1.92385571928895 \\
\hline-0.84060754774587 & -1.89206427085389 & 3.73031092412032 \\
\hline-2.31874642425413 & -1.94200828890181 & -0.91743803281245 \\
\hline 0.27476922800026 & 0.01714667699265 & 5.61496585170555 \\
\hline-2.51007387509874 & -0.08490144688638 & -3.13519272771440 \\
\hline-3.61273954479703 & -2.32976386836656 & 4.51649192694889 \\
\hline-5.02936087945261 & -2.38434104995442 & 0.05998869894529 \\
\hline-4.49998678579412 & -0.48615833818204 & 4.82441861269983 \\
\hline-5.94776234846985 & -0.54322012926355 & 0.27683891568962 \\
\hline-3.60718463679244 & -3.30178121232133 & 6.33977418312833 \\
\hline-6.06933049497534 & -3.40034220132850 & -1.40760918688374 \\
\hline-0.00677460493045 & -0.67545911751327 & 7.53880257718996 \\
\hline-3.88737961548119 & -0.79221927298443 & -4.50106849862253 \\
\hline-0.66302650524337 & 1.84514225643121 & 5.44529029199876 \\
\hline-3.13081332669211 & 1.76806889651873 & -2.48234792443077 \\
\hline 2.29735639370440 & 0.27646797662281 & 5.34580662206593 \\
\hline-0.71441225782768 & 0.13680953100476 & -4.10746490157375 \\
\hline 0.97765579342116 & -4.82393284070906 & 5.93386288016842 \\
\hline-2.09938250058474 & -4.93966130836869 & -3.69141645605925 \\
\hline-0.23354148868193 & -5.96657631529513 & 3.06150087759150 \\
\hline-1.38762913319350 & -6.00619095827553 & -0.62820938670209 \\
\hline 2.54968456509731 & -4.13042833552368 & 3.07299591765252 \\
\hline 0.84088931873594 & -4.18240000226586 & -2.31147967005004 \\
\hline 6.37489031145901 & 3.69269208293584 & 1.37657361906754 \\
\hline 5.60314049259297 & 0.96506138668567 & 3.16420917320498 \\
\hline 6.57311951932335 & 0.66709851147256 & -0.01576590443569 \\
\hline 1.65400176904995 & 3.12337103744889 & 1.80933007219188 \\
\hline 3.74311519264759 & 2.46990901715320 & -4.25342677205638 \\
\hline 3.65146188515958 & 3.77985901601689 & -6.52514451520338 \\
\hline 2.42320137080089 & 6.10293819252279 & -6.66512856747890 \\
\hline
\end{tabular}




$\begin{array}{llll}1.37320574907220 & 5.76967399506143 & -2.24854597302255 & \mathrm{c} \\ 1.28554735817179 & 7.09665996714744 & -4.51835695017343 & \mathrm{c} \\ 4.68993309363405 & 0.65652684824327 & -4.16963824264230 & \mathrm{~h} \\ 4.54195218399008 & 2.99129096516324 & -8.19157961150036 & \mathrm{~h} \\ 2.35524942899525 & 7.12646928310368 & -8.43626330534181 & \mathrm{~h} \\ 0.46949785500930 & 6.54931103767419 & -0.58286175575833 & \mathrm{~h} \\ 0.32159815844838 & 8.90056762725781 & -4.60784654746591 & \mathrm{~h}\end{array}$

\begin{tabular}{|c|c|c|}
\hline 2.30817173067670 & 6.28795967385458 & -5.81196333785828 \\
\hline 3.45066200208230 & 4.58479007075459 & -7.44709238702725 \\
\hline 4.15476264485428 & 2.21675107858358 & -6.54586579086805 \\
\hline 3.71747194863487 & 1.57145481692367 & -4.04086802692249 \\
\hline 2.55356852499274 & 3.26103653481364 & -2.37379828165006 \\
\hline 1.86816822927360 & 5.63143118515352 & -3.30125726402627 \\
\hline 2.20208017998058 & 2.49358306683442 & 0.40520975451846 \\
\hline 4.83253983896380 & 2.49632456060762 & 1.64522175664665 \\
\hline 1.39532825509550 & -0.16992009433489 & 0.44260925090786 \\
\hline-1.06678680912642 & -0.80503731196746 & 1.42105456875512 \\
\hline-0.65016437128866 & -2.63680827050947 & 3.55616167456533 \\
\hline-3.25436144685380 & -3.54347821943894 & 4.49902687328981 \\
\hline-4.94281415346250 & -4.58680463107835 & 2.42101613199380 \\
\hline-5.25168144738655 & -2.57793546554364 & 0.38989985557538 \\
\hline-2.73416270856432 & -1.62629871028416 & -0.73305841240841 \\
\hline 1.05575829832200 & -4.91319131234074 & 2.90430405520293 \\
\hline 0.59154298865083 & -1.21817758073352 & 5.76051078868679 \\
\hline-1.57082144072564 & -3.63690706308162 & -2.49545949969008 \\
\hline-3.37230730299403 & 0.70098974056318 & -2.34265926888341 \\
\hline-6.78805326125112 & -5.09955446256643 & 3.19753655026686 \\
\hline-4.14972398270355 & -6.32204540971395 & 1.62865713283046 \\
\hline-4.21545979723349 & -1.94419997471067 & 5.39334545188063 \\
\hline-6.25069048976114 & -0.96045106643028 & 1.20623394838361 \\
\hline-2.93547119547426 & -4.95069015069478 & 5.97785165761012 \\
\hline-6.41442307631636 & -3.26907763637073 & -1.17168955932187 \\
\hline 0.67296870455288 & -2.45645741046126 & 7.40960547817865 \\
\hline-4.78627602853593 & 0.18367652032113 & -3.75464190595227 \\
\hline-0.50137364476285 & 0.45135912749741 & 6.27313575595302 \\
\hline-4.16260769022325 & 2.20341119434311 & -1.17467998565709 \\
\hline 2.51572926371500 & -0.65197808643161 & 5.31197995124841 \\
\hline-1.73154890833392 & 1.42661520163501 & -3.34341428239231 \\
\hline 1.60560231851720 & -5.87311567927594 & 4.64706545521562 \\
\hline-2.70480826087863 & -3.77168123735755 & -4.21469930027552 \\
\hline 0.12748607286320 & -6.30177571324507 & 1.70701835594043 \\
\hline-1.50647808660194 & -5.51129742424464 & -1.65425009153475 \\
\hline 2.76941028169207 & -4.28265266616079 & 1. 95599347625819 \\
\hline 0.33988833575961 & -3.10398662320983 & -3.03851529285406 \\
\hline 5.75127889057897 & 4.32115703448024 & 1.36164642516836 \\
\hline 4.69598582377053 & 2.14344689465729 & 3. 66977292626150 \\
\hline 6.02401144386700 & 1.04329682490828 & 0.80068460061013 \\
\hline 0.44994840085871 & 4.25431401677717 & 1.85977084936625 \\
\hline 4.26010129222635 & -0.28142782394374 & -3.36869846913144 \\
\hline 5.04948031455557 & 0.86695464606035 & -7.79879050406845 \\
\hline 3.79007420356556 & 5.09231780852196 & -9.40054958299313 \\
\hline 0.96347982938026 & 6.99608823733674 & -2.07864383116320 \\
\hline 1.74650736646311 & 8.13860790439922 & -6.48395355560443 \\
\hline 1.31092469668663 & 6.12445974506034 & 1.96953568254592 \\
\hline
\end{tabular}




$\begin{array}{rrrr}-1.39861445741314 & 4.43268966255487 & 0.98273703624964 & \mathrm{~h} \\ 0.18569667931158 & 3.57223447748811 & 3.77696318617282 & \mathrm{~h}\end{array}$

$2 a$

\begin{tabular}{|c|c|c|}
\hline-8.16085835399132 & 5.34134688265962 & -2.27347917321545 \\
\hline-9.44270173072178 & 5.65219753422932 & 0.00000000000000 \\
\hline-8.16085835399132 & 5.34134688265962 & 2.27347917321545 \\
\hline-5.60861211974907 & 4.72046461593797 & 2.26784939961739 \\
\hline-4.30305685735886 & 4.40170999572694 & 0.00000000000000 \\
\hline-5.60861211974907 & 4.72046461593797 & -2.26784939961739 \\
\hline-1.55321196156091 & 3.68644111464993 & 0.00000000000000 \\
\hline-1.40583408191323 & 0.97215887440922 & 0.00000000000000 \\
\hline 1.15862652031901 & 0.16322388854602 & 0.00000000000000 \\
\hline 1.80012038476345 & -1.30080508035933 & 2.33482736154803 \\
\hline 3.90405873304342 & -2.94698434094425 & 1.31446761438500 \\
\hline 3.90405873304342 & -2.94698434094425 & -1.31446761438500 \\
\hline 1.80012038476345 & -1.30080508035933 & -2.33482736154803 \\
\hline 5.66260916486076 & -4.36841188559187 & -2.64617822488168 \\
\hline 7.42358930125619 & -5.80136755856017 & -1.31593412045551 \\
\hline 7.42358930125619 & -5.80136755856017 & 1.31593412045551 \\
\hline 5.66260916486076 & -4.36841188559187 & 2.64617822488168 \\
\hline-0.38052106901314 & -2.92011674546009 & 3.38725667001492 \\
\hline 2.70432307369322 & 0.53734380009499 & 4.38558047518312 \\
\hline 2.70432307369322 & 0.53734380009499 & -4.38558047518312 \\
\hline-0.38052106901314 & -2.92011674546009 & -3.38725667001492 \\
\hline 5.67951483304981 & -4.37088325668194 & 4.69408671383478 \\
\hline 5.67951483304981 & -4.37088325668194 & -4.69408671383478 \\
\hline 8.80560160037839 & -6.91668689987708 & 2.33358016486198 \\
\hline 8.80560160037839 & -6.91668689987708 & -2.33358016486198 \\
\hline 1.15820210773552 & 1.76554063478162 & -4.98384275237414 \\
\hline-1.97481256629991 & -1.73018936819506 & -3.91840189851118 \\
\hline-1.97481256629991 & -1.73018936819506 & 3.91840189851118 \\
\hline 1.15820210773552 & 1.76554063478162 & 4.98384275237414 \\
\hline 3.36294215138430 & -0.48668672013695 & -6.05250766586182 \\
\hline 0.26833201279036 & -3.93523149542609 & -5.06224261615951 \\
\hline 0.26833201279036 & -3.93523149542609 & 5.06224261615951 \\
\hline 3.36294215138430 & -0.48668672013695 & 6.05250766586182 \\
\hline 4.24194871543438 & 1.71082126081533 & -3.67809508041007 \\
\hline-1.04034924660801 & -4.31076269836047 & -2.02210560811009 \\
\hline-1.04034924660801 & -4.31076269836047 & 2.02210560811009 \\
\hline 4.24194871543438 & 1.71082126081533 & 3.67809508041007 \\
\hline-0.59589556984321 & 4.43227264524625 & 1.67159237842508 \\
\hline-4.61417426349364 & 4.48377804878023 & 4.04308242322824 \\
\hline-9.14783025356716 & 5.58797500644168 & 4.04969000981684 \\
\hline-11.43019916041301 & 6.14143490210965 & 0.00000000000000 \\
\hline-4.61417426349364 & 4.48377804878023 & -4.04308242322824 \\
\hline-9.14783025356716 & 5.58797500644168 & -4.04969000981684 \\
\hline-0.59589556984321 & 4.43227264524625 & -1.67159237842508 \\
\hline
\end{tabular}

$2 \mathrm{~b}$
$-2.37983184883796$
0.25500909198344
1.46415751144130
0.75009144502547
$-0.59377694559238$
$-0.68834229584022$
1. 87706977274487
$-2.03179832298410$ . 


\begin{tabular}{|c|c|c|}
\hline 3.58026873051021 & 1.43812395743039 & 2.65311380413928 \\
\hline 3.18948708276426 & -0.76760546128050 & 4.02497118255324 \\
\hline 5.69320494341565 & 2.92858861447004 & 3.10534216255603 \\
\hline 4.90475253776626 & -1.51656710861673 & 5.86520940048042 \\
\hline 6.01616476633987 & 4.64548421572061 & 2.03700936224531 \\
\hline 4.61517202040367 & -3.23876030363323 & 6.93514257333876 \\
\hline 7.41153798111880 & 2.18914654644291 & 4.95514478286833 \\
\hline 7.02054752405064 & -0.02289190208251 & 6.32719471363228 \\
\hline 9.06631137983583 & 3.33609020924078 & 5.32385995784689 \\
\hline 8.37272406135284 & -0.58669620275215 & 7.75664448949239 \\
\hline 1.06770951303294 & -4.86425603361001 & 2.71701232929296 \\
\hline-1.30404597266990 & -1.65309900065541 & 5.27321502619098 \\
\hline-0.27885680815963 & 4.03729962100851 & 1.65737880682220 \\
\hline 2.45935317374743 & 2.45839797843987 & -1.87866375274043 \\
\hline-0.70070685226686 & -5.67347184684499 & 2.02743283409748 \\
\hline-3.10860529063509 & -2.41391968554490 & 4.63869826167802 \\
\hline-1.87332005615497 & 4.24804421955572 & 0.37282786631054 \\
\hline 0.90363841407242 & 2.63703228127350 & -3.21599161787828 \\
\hline 1.58263882438265 & -5.87863236182434 & 4.43956090241783 \\
\hline-0.74698990587945 & -2.64130636551396 & 6.99662710359456 \\
\hline 0.78282800379806 & 5.80626177655742 & 1. 67105508509281 \\
\hline 3.50764742214007 & 4.23705185507452 & -1.88727161134840 \\
\hline 2.53203610430169 & -5.17787655272268 & 1.30281369461263 \\
\hline-1.56859826445432 & 0.33293307968041 & 5.74187660516493 \\
\hline-1.00881654606016 & 3.72207622928779 & 3.55547839772583 \\
\hline 3.70991215609639 & 0.95393133160571 & -2.52351372403635 \\
\hline-3.06653070232025 & -2.07645864741622 & -1.92071834835554 \\
\hline-5.61259532064068 & -3.28816888910732 & -1.31496133697885 \\
\hline-3.18061248697280 & -0.50403813325266 & -4.31359261644142 \\
\hline-1.63169744067509 & -3.53659087094807 & -2.16248527938263 \\
\hline-6.27255115050267 & -4.40579463226067 & -2.91744064365697 \\
\hline-5.45558558466134 & -4.51683133940974 & 0.33337534271222 \\
\hline-7.02894889071076 & -1.84232132179088 & -0.91746476010320 \\
\hline-1.95669548734534 & -1.31353664743076 & -6.49984857273251 \\
\hline-2.10309698273687 & 0.08129631236340 & -8.72504480085276 \\
\hline-3.47575026021017 & 2.31863290373840 & -8.78519430205978 \\
\hline-4.56100729435131 & 1.74387877776559 & -4.39720373680320 \\
\hline-4.70260241263524 & 3.14809079320794 & -6.61028028032517 \\
\hline-0.86392538578346 & -3.04653253921562 & -6.45891869575306 \\
\hline-1.13357436682376 & -0.57469311019294 & -10.40448657893483 \\
\hline-3.58670242632387 & 3.41384870420380 & -10.51062145989224 \\
\hline-5.51032304148510 & 2.40777438167130 & -2.70943398777229 \\
\hline-5.77322190828302 & 4.89291295903941 & -6.64186078147789 \\
\hline
\end{tabular}

$2 c$

$-5.25184813573094$

$-4.29887707141103$

$-2.79453163524581$

$-2.24391865550695$

$-3.18053716619550$

$-4.70160873741813$

$-2.68995152530743$

$-5.18994797394386$

$-2.03589004259406$

0.52489174622948

1. 85822018188348
3. 69190385018375

3. 38956949172821

1. 30949883888128

$-0.45331397080467$

$-0.17377616312899$

1. 92907272261169

$-2.10233777109177$

$-3.40743653733875$

$-0.70089570954889$

$-0.76539529915472$

1. 72478235545532 $\begin{array}{rl}-5.61225873962020 & c \\ -8.04495783809987 & c \\ -8.58118214559458 & c \\ -6.70480906191546 & c \\ -4.25661718941041 & c \\ -3.74940152928291 & c \\ -2.14558255895650 & c \\ -1.46115454038872 & c \\ 0.16630677787637 & \circ \\ 0.95660962277537 & n \\ 0.63869347953234 & c\end{array}$ 


\begin{tabular}{|c|c|c|}
\hline 3.75944750041167 & 1.54505699269118 & 2.76570477334678 \\
\hline 3.11054749858495 & -0.35814119700718 & 4.45434212502709 \\
\hline 0.69761433889288 & -1.65707672536748 & 3.64710309615853 \\
\hline 4.58958331002465 & -0.82279424030222 & 6.57267263985380 \\
\hline 6.73074475629288 & 0.64824317465236 & 6.98776112038460 \\
\hline 7.38175822676323 & 2.55603804760511 & 5.29525225769649 \\
\hline 5.89874573548189 & 3.01146570363187 & 3.17063961536764 \\
\hline 0.16517636307034 & 4.07025342724372 & 0.96393366314184 \\
\hline 3.13210409460246 & 1.81499584557534 & -1.96225852014752 \\
\hline 0.92017468811465 & -4.54374612145524 & 3.71344231287281 \\
\hline-1.53049691945817 & -0.83932851385011 & 5.33917544087095 \\
\hline 6.42143906938522 & 4.49334273849655 & 1.85747479072325 \\
\hline 4.09894392377087 & -2.30850105546636 & 7.89403540689192 \\
\hline 9.05466280655756 & 3.68684881552576 & 5.63050131816601 \\
\hline 7.89997918900798 & 0.30321660419915 & 8.63197799811947 \\
\hline-0.81993060146427 & -5.42101781696839 & 3.03818220997766 \\
\hline-3.29667805393992 & -1.64641358198301 & 4.65591294816464 \\
\hline-1.26162020202288 & 4.15145401849761 & -0.51547488123390 \\
\hline 1.72394222277720 & 1.80595054216839 & -3.46624124858691 \\
\hline 1.23179304937781 & -5.20124069956893 & 5.64489898929372 \\
\hline-1.20422259319931 & -1.51056655720190 & 7.26278449436665 \\
\hline 1.33000062212416 & 5.76838701970224 & 0.83238510691952 \\
\hline 4.24602407725106 & 3.54129440645328 & -2.16334440399040 \\
\hline 2.48836108725309 & -5.18398944498095 & 2.54173779706807 \\
\hline-1.73710769987046 & 1.20626246856086 & 5.41207661442743 \\
\hline-0.78230541417233 & 4.09535306659164 & 2.79019543166479 \\
\hline 4.38534115356088 & 0.19860858987841 & -2.21128120849374 \\
\hline-0.72967313132393 & -4.08748927466903 & -2.85311625441752 \\
\hline-6.00891245854142 & -4.31705776670328 & -3.12109614496790 \\
\hline-4.87223664051335 & -4.83585789285758 & -0.00784050228293 \\
\hline-6.55070979379162 & -2.03828299922033 & -0.74254904590978 \\
\hline-1.06152067201424 & -2.05004490725958 & -7.18127265654042 \\
\hline-2.03667335428498 & 1.04765853669034 & -10.46497795345981 \\
\hline-4.72711633224411 & 4.76141159970951 & -9.50215745334480 \\
\hline-5.44157843781521 & 2.19681048580942 & -1.86194983649649 \\
\hline-6.42920025310660 & 5.30614118952076 & -5.16493162382023 \\
\hline-1.44272251853520 & -5.21011499682549 & -4.42884429278979 \\
\hline 1.07355895132302 & -3.26065000971156 & -3.38792405611140 \\
\hline-0.40323857308946 & -5.35815127959730 & -1.27257634482574 \\
\hline
\end{tabular}

$3 b$

$$
\begin{array}{r}
-5.00204446807778 \\
-5.78523446952705 \\
-8.17284797914824 \\
-9.82153015006235 \\
-9.06177950947560 \\
-6.66939178373788 \\
-4.50723913797414 \\
-8.74771119842569 \\
-10.32560662057428 \\
-6.08542177838440 \\
-2.44487994737631 \\
-0.57909559808858 \\
1.61923987379064 \\
1.41805447614541 \\
3.89714879119246
\end{array}
$$

$\begin{array}{rc}0.81170400058392 & c \\ -0.15749403396503 & c \\ -1.22049643558379 & c \\ -1.31885515583734 & c \\ -0.35027496228167 & c \\ 0.70406842280225 & c \\ -0.09653876018893 & h \\ -1.97389825862471 & h \\ -0.42682467970044 & h \\ 1.43827731593757 & h \\ 2.08279067423669 & c \\ 0.80766538027502 & o \\ -0.06358444235611 & n \\ -2.87488075473265 & c \\ 0.99182726651825 & c\end{array}$




\begin{abstract}
0.23321863247608
$-0.84395940721330$

2. 14433294765616

1. 85670902958212

3. 78653936531283

2.01027124588580

0.41036258768004

1. 38776196450532

$-1.46581347513483$

2. 59699266879407

4. 28949530171120

1.82308222215174

$-1.75297045207407$

$-2.75477083310236$

$-3.04523206531450$

$-3.87814534664235$

$-4.36832261630480$

$-4.72789100268883$

$-0.61718342237215$

$-1.52557066060421$

$-1.70096050451067$

2.09496451308476

$-0.58264200415787$

2.44435316138081

$-0.30945242096939$

0.68727192974796

0.12206368693901

$-2.33382177164991$

2. 09279172258728

3. 14496704646172

3. 14242668809407

3. 15214180566493

$-1.24042096696842$

$-4.73344669845843$
\end{abstract}

6.28521260470890

7.91814269003550

6.48345229995732

3. 90701124965978

4. 02982400461851

3. 79127971249532

6. 25641982738947

7.95239439768416

6.29913756533761

$-0.69057449697468$

$-0.42531653605728$

$-2.55727301979269$

0.80566742876419

$-0.77252921147479$

2. 39913563745332

3. 94229078409044

4. 31982977029558

2. 14883754614688

3.95398962811307

5.68220795693797

2.40016811441890

3. 87274384197060

$-11.68013505689636$

$-1.84596449284629$

$-2.65154442986316$

$-4.21842273759672$

$-0.93065788793820$

$-3.02642435844063$

3. 69199314939241

2. 28375261471134

5.59085266014190

$-0.63970386936014$

0.26240967307320

5.43013243956838

$\begin{array}{rl}-0.14088917784910 & \mathrm{c} \\ 0.52478572505003 & \mathrm{~h} \\ 0.61309294886377 & \mathrm{~h} \\ -3.81592089016524 & \mathrm{c} \\ -3.07963003505974 & \mathrm{~h} \\ -5.87277238226378 & \mathrm{~h} \\ -3.01076336328434 & \mathrm{c} \\ -3.67344983303404 & \mathrm{~h} \\ -3.87340882091476 & \mathrm{~h} \\ -3.39128807838824 & \mathrm{c} \\ -2.24327317602943 & \mathrm{~h} \\ -3.01437866788534 & \mathrm{~h} \\ -4.37638303022189 & \mathrm{c} \\ -3.51794823932493 & \mathrm{~h} \\ -4.50978980830203 & \mathrm{~h} \\ 0.52620149060072 & \mathrm{c} \\ -1.43380559395870 & \mathrm{~h} \\ 1.06943285240059 & \mathrm{~h} \\ 3.89619580077104 & \mathrm{c} \\ 4.57834537150778 & \mathrm{~h} \\ 4.70593668620195 & \mathrm{~h} \\ 4.88663280700053 & \mathrm{c} \\ -2.14908388596998 & \mathrm{~h} \\ 1.95811038598811 & \mathrm{~h} \\ 4.86027235954778 & \mathrm{c} \\ 5.75899580413013 & \mathrm{~h} \\ 5.89968778838514 & \mathrm{~h} \\ 4.99626999422259 & \mathrm{~h} \\ 6.94367063265826 & \mathrm{~h} \\ 4.10325278954482 & \mathrm{~h} \\ 4.43504653533708 & \mathrm{~h} \\ -5.37887655508137 & \mathrm{~h} \\ -6.30063536545431 & \mathrm{~h} \\ 1.67288135389389 & \mathrm{~h} \\ -10\end{array}$

$-0.14088917784910$

$\mathrm{h}$ h (1) $\mathrm{h}$ h $\mathrm{h}$ h h

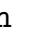
.

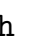
rate . (

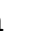
(

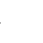
h c h

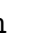

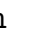
.

$4 b$
0.30291708007895
$-2.11533497448861$
$-2.53015054326548$
$-0.53571709078435$
1.87990718903832
2. 28990967593026
$-3.67357020565767$
$-4.41822705371004$
3. 44989084212992
4.18389379978047
0.76725043692522
$-0.86217960438507$
0.42550184551331
0.64198937267698
$-0.86565873550384$
0.21982067016660
$-0.85568861615382$
2.16906385828636
1. 69416473859132
3. 65692614846422

$-5.31485302013479$

$-6.12253268346878$

$-8.59444915166327$

$-10.30240387532922$

$-9.51782782176051$

$-7.04248369630672$

$-4.79999078629750$

$-9.18833956712772$

$-10.82825722648684$

$-6.44215134801725$

$-2.65522782486440$

$-0.89368495124656$

1. 21453966656635

0.70786195238986

3. 58676736088513

5.84563442277148

7.52745600797570

6.17659345488793

3. 10152322013169

3. 36282250181823
$0.98675413851497 \quad \mathrm{c}$

$0.31509429851728 \quad \mathrm{c}$

$-0.47389249533922 \quad \mathrm{c}$

$-0.59199316367730 \quad \mathrm{c}$

$0.08084584310117 \quad \mathrm{c}$

$0.86213246937001 \mathrm{c}$

$0.39310661007506 \mathrm{~h}$

$-0.99775811658244 \mathrm{~h}$

$-0.01458904091849 h$

$1.36667389517805 \mathrm{~h}$

$1.96429295288710 \quad \mathrm{c}$

0.66326379434373 o

$-0.50947864508218 \mathrm{n}$

$-3.29973307774998 \quad \mathrm{c}$

$0.38634917816541 \quad \mathrm{c}$

$-1.10509928894363 \mathrm{c}$

$-0.58737362081394 \mathrm{~h}$

$-0.51168652934598 \mathrm{~h}$

-4.58199661811399 c

$-3.98092131531580 \mathrm{~h}$ 


$\begin{array}{rrrr}1.73657647042821 & 2.75489216316795 & -6.61817112819761 & \mathrm{~h} \\ 0.21323658235235 & 5.48088999554169 & -3.95761058702690 & \mathrm{c} \\ 1.07911632689415 & 7.11791579306811 & -4.87494025552474 & \mathrm{~h} \\ -1.71573699938005 & 5.36490393437798 & -4.68699678703590 & \mathrm{~h} \\ 2.61632194205817 & -1.39013373495746 & -3.67789673583049 & \mathrm{c} \\ 4.34939363201099 & -0.95338632465525 & -2.64928437667191 & \mathrm{~h} \\ 1.92346979714159 & -3.22790218637572 & -3.07033060926164 & \mathrm{~h} \\ -1.82075658508964 & -0.14625463708473 & -4.60877942685422 & \mathrm{c} \\ -2.77871936145111 & -1.58003533094099 & -3.48807564108762 & \mathrm{~h} \\ -3.13323346949637 & 1.39933681382167 & -4.95279152039770 & \mathrm{~h} \\ -3.76417969396498 & 3.48483572284724 & 0.07692156889828 & \mathrm{c} \\ -4.32713521434869 & 3.21611971123029 & -1.87849625367872 & \mathrm{~h} \\ -4.56023819081355 & 1.93932735281784 & 1.17941130168272 & \mathrm{~h} \\ -0.23020928650846 & 3.91301046172415 & 3.26609386969667 & \mathrm{c} \\ -1.44547446514211 & 6.24440682012316 & 4.50127553517283 & \mathrm{c} \\ -1.06553007244054 & 2.26704477384609 & 4.18119516543682 & \mathrm{~h} \\ 2.60884063386554 & 3.91542530662205 & 3.85908456247084 & \mathrm{c} \\ -0.86038094104175 & -12.22661251666239 & -1.20906971516390 & \mathrm{~h} \\ 2.72002877553245 & -2.12023880449692 & 1.58494289998063 & \mathrm{~h} \\ 0.27814017169334 & -2.57838395866461 & 4.81623962898062 & \mathrm{C} \\ 1.32843808416764 & -4.08996901582291 & 5.74798464839585 & \mathrm{~h} \\ 0.86390943112035 & -0.78684177127465 & 5.63858443552672 & \mathrm{~h} \\ -1.72798134669332 & -2.87038189790989 & 5.20019454349907 & \mathrm{~h} \\ 2.90334568702970 & 3.67211515235662 & 5.88882540882619 & \mathrm{~h} \\ 3.60198406164865 & 2.40880330572511 & 2.87202294337870 & \mathrm{~h} \\ 3.49590802117871 & 5.70640016660245 & 3.33971073000084 & \mathrm{~h} \\ 3.07858628743752 & -1.53825681744838 & -5.68434429186740 & \mathrm{~h} \\ -1.35983138990349 & -0.97571115821155 & -6.44169017533105 & \mathrm{~h} \\ -4.61993669418801 & 5.24498272184526 & 0.71593535708208 & \mathrm{~h} \\ -0.94390689311449 & 6.29203135826475 & 6.50356631822717 & \mathrm{~h} \\ -0.76710097999459 & 8.00777154892598 & 3.66876638782419 & \mathrm{~h} \\ -3.50165315462110 & 6.23289841687443 & 4.39373093057971 & \mathrm{~h} \\ ---------------------------------------------------------- \\ -10\end{array}$

$5 b$

$\begin{array}{rrrr}0.50202070548010 & -5.49014308861803 & 0.52945782620378 & \mathrm{c} \\ -1.95954335081402 & -6.22203508450499 & -0.06348519173543 & \mathrm{c} \\ -2.45666418374717 & -8.63507954408151 & -0.97873533909768 & \mathrm{c} \\ -0.50148429916719 & -10.36103282837972 & -1.30140742180358 & \mathrm{c} \\ 1.95768285355516 & -9.65454288942744 & -0.70222590674636 & \mathrm{c} \\ 2.44877739460270 & -7.23871951634599 & 0.20630240773009 & \mathrm{c} \\ -3.48956716480177 & -4.88684659243028 & 0.17997807209308 & \mathrm{~h} \\ -4.37905200595249 & -9.16837434136528 & -1.43905501222751 & \mathrm{~h} \\ 3.49768725396642 & -10.97996207866071 & -0.95275448352552 & \mathrm{~h} \\ 4.37562866713574 & -6.70035078040823 & 0.65372568388966 & \mathrm{~h} \\ 1.05637626930999 & -2.90933682426268 & 1.67110298044603 & \mathrm{c} \\ -0.64614350875793 & -1.03287876589534 & 0.64623864363425 & \mathrm{O} \\ 0.55609766634081 & 1.11046247784280 & -0.59764293795537 & \mathrm{n} \\ 0.79804162438042 & 0.52642767455048 & -3.38734852779884 & \mathrm{c} \\ -0.87642531580330 & 3.45898394481140 & 0.19890380473712 & \mathrm{c} \\ 0.10820502942400 & 5.69329071927616 & -1.41797173899604 & \mathrm{c} \\ -1.07318758552387 & 7.32863919483263 & -1.00954206559908 & \mathrm{~h} \\ 2.02434129664738 & 6.17496501978325 & -0.81851357224017 & \mathrm{~h} \\ 1.75924687548859 & 2.90684611858730 & -4.75760666675981 & \mathrm{c} \\ 3.69999091320577 & 3.28133312486210 & -4.14463290977455 & \mathrm{~h} \\ 1.84560498455257 & 2.47241451819665 & -6.77514356214225 & \mathrm{~h} \\ 0.16314024105817 & 5.22496397845672 & -4.24956311870080 & \mathrm{c}\end{array}$




$\begin{array}{rrrr}0.96393532336956 & 6.87376863405570 & -5.20488097422627 & \mathrm{~h} \\ -1.74307853208576 & 4.99336549489001 & -5.00927819335701 & \mathrm{~h} \\ 2.87458474276830 & -1.47715804635632 & -3.75006104376367 & \mathrm{c} \\ 4.57582127620451 & -0.96326973378862 & -2.70451106329861 & \mathrm{~h} \\ 2.27508235916814 & -3.35693365323284 & -3.18479128269665 & \mathrm{~h} \\ -1.62915238046400 & -0.46617725731388 & -4.65708700020299 & \mathrm{c} \\ -2.47069625920366 & -1.97230441430303 & -3.53976320750205 & \mathrm{~h} \\ -3.04590940232986 & 0.99458528630273 & -4.94634805200599 & \mathrm{~h} \\ -3.75948246389564 & 3.22983609122206 & -0.18462109393985 & \mathrm{c} \\ -4.27670855500563 & 3.34556643963963 & -2.16972558843210 & \mathrm{~h} \\ -4.47959433119229 & 1.45461624843885 & 0.55686949257913 & \mathrm{~h} \\ -0.29829882562156 & 4.11734081508205 & 3.10608492740062 & \mathrm{c} \\ -0.79307417458163 & 6.94405563716849 & 3.68004786010037 & \mathrm{c} \\ -2.03845320718642 & 2.66399514903508 & 4.94631581737026 & \mathrm{c} \\ 2.49138322443628 & 3.62084448019142 & 3.75830333742408 & \mathrm{c} \\ -0.88981567375790 & -12.23886777479720 & -2.01657308335363 & \mathrm{~h} \\ 2.98356925367343 & -2.34868753388877 & 1.21258008053643 & \mathrm{~h} \\ 0.78707833721260 & -3.12619168700307 & 4.54904068859920 & \mathrm{c} \\ 1.96882078607866 & -4.67804053934463 & 5.22137738742705 & \mathrm{~h} \\ 1.36231025277139 & -1.40790224637831 & 5.51614576434489 & \mathrm{~h} \\ -1.16298420797195 & -3.56036929950895 & 5.06190304891092 & \mathrm{~h} \\ 2.85885131661949 & 4.20796969172438 & 5.70463884484023 & \mathrm{~h} \\ 3.01105526733304 & 1.64595182862841 & 3.56987139219036 & \mathrm{~h} \\ 3.75886960795912 & 4.69784673674982 & 2.53873963125378 & \mathrm{~h} \\ 3.36206948945787 & -1.57474567916496 & -5.75356854228414 & \mathrm{~h} \\ -1.15423571978850 & -1.24235181620117 & -6.50994067356310 & \mathrm{~h} \\ -4.73277842979786 & 4.76940182431639 & 0.77991409161054 & \mathrm{~h} \\ -0.67265182044809 & 7.22914043194714 & 5.72116108320477 & \mathrm{~h} \\ 0.58737610062124 & 8.19889553372079 & 2.80828861986928 & \mathrm{~h} \\ -2.67732313663217 & 7.54910669825817 & 3.09620063209733 & \mathrm{~h} \\ -2.18898371322951 & 0.67374809428111 & 4.49959687472555 & \mathrm{~h} \\ -3.94349952051628 & 3.45437751015105 & 4.94177468703053 & \mathrm{~h} \\ -1.31486134454518 & 2.84956261865907 & 6.87221457347972 & \mathrm{~h} \\ - & - & & \end{array}$

$6 b$

$\begin{array}{rrrr}-1.04478891602718 & -0.85212539494309 & 1.16685607710396 & \mathrm{o} \\ 0.35940710020708 & 1.32670295786660 & 0.29370796470290 & \mathrm{n} \\ 0.76231010721671 & 1.11233378268423 & -2.52375782207216 & \mathrm{c} \\ -0.89721025695150 & 3.63209399338489 & 1.39538388250940 & \mathrm{c} \\ 0.30913221762578 & 6.00384191793584 & 0.20476887083716 & \mathrm{c} \\ -0.71961296593066 & 7.65453233640855 & 0.89453146901169 & \mathrm{~h} \\ 2.24639593669440 & 6.19470226111059 & 0.89177494145167 & \mathrm{~h} \\ 1.86725667528085 & 3.63186042767217 & -3.48856674921238 & \mathrm{c} \\ 3.79652838220096 & 3.83471345221085 & -2.78567041030094 & \mathrm{~h} \\ 2.00484659834820 & 3.51838676004296 & -5.54517627782067 & \mathrm{~h} \\ 0.38972572860507 & 5.95561739702334 & -2.66777422669824 & \mathrm{C} \\ 1.31742543229818 & 7.66258482323624 & -3.37325082347935 & \mathrm{~h} \\ -1.51245703715982 & 5.96215151024089 & -3.47118587298472 & \mathrm{~h} \\ -0.37040783911661 & 3.69321082906818 & 4.27664380597918 & \mathrm{C} \\ -1.09957007832282 & 5.48869248317696 & 4.98791354343703 & \mathrm{~h} \\ -1.51693896016466 & 2.23416817227845 & 5.17403105720577 & \mathrm{~h} \\ 2.37344094058666 & 3.43249242613879 & 5.14130937982835 & \mathrm{c} \\ 3.55145772013154 & 4.99251474164244 & 4.48447393185787 & \mathrm{~h} \\ 2.46017479790313 & 3.43798054923258 & 7.20476757450380 & \mathrm{~h} \\ 3.23447549155616 & 1.68846416318337 & 4.47083090557075 & \mathrm{~h} \\ 2.74368069058195 & -0.99232797796931 & -3.00778205463193 & \mathrm{c}\end{array}$




\begin{tabular}{|c|c|c|}
\hline 1.92525577736274 & -2.79877392451895 & -2.45299976668735 \\
\hline 3.02114232737525 & -1.11710884173790 & -5.04832371411565 \\
\hline 5.34278641984648 & -0.68597090498398 & -1.77397446025479 \\
\hline 5.19817575034372 & -0.19179695050141 & 0.21990136104138 \\
\hline 6.40164941674776 & -2.45190573651219 & -1.92309727638547 \\
\hline 6.47545311751999 & 0.77267160801949 & -2.69241661538202 \\
\hline-3.81585058910856 & 3.65405328505130 & 0.98144901435942 \\
\hline-4.52508109375734 & 1.77312447809508 & 1.42863128786751 \\
\hline-4.20759582883759 & 3.95848629664769 & -1.01563999085364 \\
\hline-5.34428050408735 & 5.60453304713447 & 2.48277311691405 \\
\hline-4.67747420030811 & 7.53625296435561 & 2.18755546610532 \\
\hline-7.31670417696735 & 5.54721352069051 & 1.87482333886984 \\
\hline-5.32364257103070 & 5.23444992340777 & 4.50989641005045 \\
\hline-1.71026954716497 & 0.44798793566660 & -3.97829188273567 \\
\hline-2.66765228043369 & -1.04667677936509 & -2.93548081000372 \\
\hline-2.96668074426878 & 2.07755966697036 & -3.93306826844366 \\
\hline-1.44166869149856 & -0.37574871468748 & -6.74266359557045 \\
\hline-0.51825899039939 & -2.20771298043376 & -6.93290370564650 \\
\hline-3.31548395447561 & -0.54871427186345 & -7.59281554369094 \\
\hline-0.38592025094361 & 0.98664225771347 & -7.88010798825437 \\
\hline 0.20954186877086 & -5.27781182676621 & 1.22090006208974 \\
\hline-2.05324742653554 & -6.09145388813523 & 0.13818058132289 \\
\hline-2.23591165087539 & -8.47562696775777 & -0.94980679492821 \\
\hline-0.16282961167990 & -10.09249261561122 & -0.95893672345896 \\
\hline 2.09641983311913 & -9.30425550136798 & 0.12535434640753 \\
\hline 2.27552045183779 & -6.91418865653303 & 1.20450558369402 \\
\hline-3.67102837835179 & -4.83961993565556 & 0.13076602659609 \\
\hline-4.00366358421043 & -9.07397723662337 & -1.79184200568268 \\
\hline 3.72555888695405 & -10.54406472911648 & 0.12165872928597 \\
\hline 4.05030395260764 & -6.30863239328525 & 2.03192451004146 \\
\hline 0.40257719605837 & -2.72518352060922 & 2.52332843433760 \\
\hline-0.30664119083521 & -11.94905483315009 & -1.80817607675768 \\
\hline 2.37169016235318 & -2.12738046793104 & 2.55115897053854 \\
\hline-0.58749581676602 & -2.95517403547188 & 5.23297332009254 \\
\hline 0.33659470703241 & -4.53213363463336 & 6.18959931550303 \\
\hline-0.23353505288425 & -1.24415329785526 & 6.31744294879190 \\
\hline-2.61702549807250 & -3.32595395027055 & 5.21389322814310 \\
\hline
\end{tabular}

$7 \mathrm{~b}$

$\begin{array}{rrrr}-0.66887738632813 & -1.25979276545696 & 1.20746866214909 & \mathrm{o} \\ 0.59189559871161 & 1.02605783324529 & 0.35899161416620 & \mathrm{~h} \\ 1.06411049558933 & 0.93704283231118 & -2.49205610393930 & \mathrm{c} \\ -0.75887194944017 & 3.24381672439926 & 1.57528942175571 & \mathrm{c} \\ 0.27421575076623 & 5.71829372373686 & 0.38760632568317 & \mathrm{c} \\ -0.93394055210112 & 7.27736453306925 & 0.96518242993329 & \mathrm{~h} \\ 2.12010243575421 & 6.09357182955971 & 1.22674551150441 & \mathrm{~h} \\ 2.32016622635416 & 3.48442596996935 & -3.13443351888779 & \mathrm{c} \\ 4.07601231539758 & 3.65363718679325 & -2.06777220425169 & \mathrm{~h} \\ 2.84244213976399 & 3.49702239330686 & -5.12538379264315 & \mathrm{~h} \\ 0.66298013829273 & 5.72798352456672 & -2.45735571737898 & \mathrm{c} \\ 1.59324805135080 & 7.49150711399562 & -3.00590071439171 & \mathrm{~h} \\ -1.12810517697401 & 5.67713208645195 & -3.47775881043803 & \mathrm{~h} \\ 0.02558124717450 & 3.33483412661420 & 4.44885248544619 & \mathrm{c} \\ -0.91209058936430 & 1.74638050753134 & 5.35012562265721 & \mathrm{~h} \\ -0.89056557270438 & 5.72804307538354 & 5.82779984476466 & \mathrm{c} \\ -2.88539325628536 & 6.12984339100285 & 5.53215919167192 & \mathrm{~h}\end{array}$




\begin{tabular}{|c|c|c|}
\hline-0.60758335371417 & 5.49511282357431 & 7.85998263287119 \\
\hline 0.17957581268345 & 7.39682961325513 & 5.25654904644210 \\
\hline 2.86421680162619 & 3.04199959699825 & 4.96580261043654 \\
\hline 3.17830138405043 & 2.92043099981769 & 7.00442130462997 \\
\hline 3.65248330760885 & 1.35917762891873 & 4.09364878062480 \\
\hline 3.95349092399170 & 4.66055608941826 & 4.29212916276317 \\
\hline 3.03721169632411 & -1.23910754399626 & -2.93287926240123 \\
\hline 2.18598102307859 & -2.93877748407499 & -2.14882350987946 \\
\hline 5.57348893736253 & -0.80482857367552 & -1.57427933081838 \\
\hline 5.33938225987677 & -0.00859494784528 & 0.30610495572717 \\
\hline 6.58469397492929 & -2.59705403217681 & -1.40210942357860 \\
\hline 6.79501107456978 & 0.46638165043321 & -2.64925802586860 \\
\hline 3.65344064557226 & -1.86498510825857 & -5.70293949613264 \\
\hline 5.14834451142201 & -3.28926576212459 & -5.73924325602487 \\
\hline 2.05801578885538 & -2.64563924750149 & -6.73540632826856 \\
\hline 4.35817612530632 & -0.22566343020579 & -6.74038086550248 \\
\hline-3.77210246046268 & 2.98740896039340 & 1.31881968455337 \\
\hline-4.06155005406059 & 1.69998183331112 & -0.25064908206515 \\
\hline-5.16323225500172 & 1.71110433131208 & 3.53456609365964 \\
\hline-5.22394443688463 & 2.87274023630924 & 5.23666947644804 \\
\hline-7.12199486118038 & 1.37184194671378 & 2.97121731006137 \\
\hline-4.33760930613273 & -0.10674731274899 & 4.01373075504254 \\
\hline-5.20405417598247 & 5.42207308055859 & 0.62910426855161 \\
\hline-5.10037378084598 & 6.85510022933850 & 2.11008433823247 \\
\hline-4.53846258860302 & 6.28148508794592 & -1.12050945944533 \\
\hline-7.20298474075358 & 4.97552571712422 & 0.36245868863391 \\
\hline-1.41623702422069 & 0.62740830951843 & -4.16090767910296 \\
\hline-2.77344336979114 & 1.92678778677536 & -3.32869898300096 \\
\hline-1.23878569772556 & 1.50889621630109 & -6.93899824811734 \\
\hline 0.00412197510818 & 0.35034801591046 & -8.09575765010479 \\
\hline-3.12485838816548 & 1.38688716705607 & -7.77272569994326 \\
\hline-0.63026735759151 & 3.46641431955043 & -7.12824274641032 \\
\hline-2.66865861052679 & -1.98955069434664 & -4.09254478665932 \\
\hline-1.46741876044816 & -3.46367699597388 & -4.88882196629445 \\
\hline-3.15626742191573 & -2.55217550408296 & -2.18295912820629 \\
\hline-4.40075810550969 & -1.94573608246832 & -5.21884983454197 \\
\hline 0.60011316841680 & -5.67665088222996 & 1. 39225792922460 \\
\hline-1.78755283506354 & -6.69388666361628 & 0.91729686659420 \\
\hline-2.04566709425516 & -9.12805734258425 & -0.03177550708440 \\
\hline 0.08172420670587 & -10.60106451527927 & -0.50068624581878 \\
\hline 2.46726521622854 & -9.61766149693688 & -0.01410851521056 \\
\hline 2.71766009305304 & -7.17368996634555 & 0.92328024688408 \\
\hline-3.45683979747331 & -5.56815147879145 & 1.28030398427452 \\
\hline-3.91344477546048 & -9.88049702979239 & -0.40127815564335 \\
\hline 4.13734126613873 & -10.74670326369295 & -0.37071904097844 \\
\hline 4.58712553037486 & -6.42104059821626 & 1.29335974456705 \\
\hline 0.89762938403318 & -3.06667882727101 & 2.55568868303921 \\
\hline-0.12101226174160 & -12.49969056496403 & -1.23745649885391 \\
\hline 2.85766733153261 & -2.47549972265837 & 2.38067101344867 \\
\hline 0.18691571206117 & -3.21497642763485 & 5.35789046419490 \\
\hline 1.23682066894934 & -4.75274618766919 & 6.24744031248550 \\
\hline 0.62860917863070 & -1.47849923057803 & 6.36252451923416 \\
\hline-1.82261440093728 & -3.62435880927351 & 5.57544560553076 \\
\hline
\end{tabular}

$8 \mathrm{~b}$

$\begin{array}{llll}1.94229052822564 & -5.63917903252380 & 1.87232144510422 & \mathrm{C}\end{array}$ 


\begin{tabular}{|c|c|c|}
\hline 0.45049441153005 & -5.96934521574522 & 4.01981179889990 \\
\hline-0.09710876350597 & -8.37777366103866 & 4.91919719640681 \\
\hline 0.85491368684259 & -10.50181943812812 & 3.70180871578356 \\
\hline 2.36388483330146 & -10.19642500147906 & 1.57341764164212 \\
\hline 2.89662305558446 & -7.78699400323309 & 0.67301322301566 \\
\hline-0.30191273991851 & -4.33894818437084 & 4.98900083075604 \\
\hline-1.27079601949923 & -8.59076311087619 & 6.58316590312424 \\
\hline 3.12083631883799 & -11.83318355280136 & 0.60444839808134 \\
\hline 4.07510411119094 & -7.57913648033502 & -0.99077073688676 \\
\hline 2.72436553373268 & -3.03530846019148 & 0.92135787155929 \\
\hline 0.54308488071983 & -1.39235693850676 & 1.23193315080655 \\
\hline 0.26397224177352 & 0.91571130033120 & -0.25039269490262 \\
\hline-0.76584780401660 & 0.54071087990517 & -3.02904381584426 \\
\hline-0.57329796726080 & 3.05134168150884 & 1.59693995563659 \\
\hline-1.10650219922128 & 5.42805770758751 & -0.02601886198889 \\
\hline-1.97888420503701 & 6.82769867870133 & 1.20154596792753 \\
\hline 0.69976404661137 & 6.23617576827591 & -0.55817533726775 \\
\hline-2.85830082011412 & 2.53187857566599 & -3.54037804640113 \\
\hline-3.09162568514654 & 2.69584258940996 & -5.57608573876689 \\
\hline-4.61674488576461 & 1.72953831634436 & -2.87401500531910 \\
\hline-2.68058046838933 & 5.19060665591804 & -2.43384302692244 \\
\hline-1.91264943728675 & 6.50609477760129 & -3.81883901296990 \\
\hline-4.60828047137338 & 5.83753691913815 & -2.10153732514329 \\
\hline 1.57751260648550 & 1.09755482114806 & -5.00036002469427 \\
\hline 0.91054172122504 & 0.65398678001590 & -7.81766289540098 \\
\hline 2.41563316891610 & 3.89416170159772 & -4.92920666101023 \\
\hline 4.01985991828150 & -0.41092547847299 & -4.50544955346047 \\
\hline-2.22249883282040 & -2.18648329481469 & -3.63827460399809 \\
\hline-4.10153552737624 & -1.96243886397822 & -5.90552142003343 \\
\hline-0.52795712960992 & -4.45750032183144 & -4.33467051415546 \\
\hline-3.91026914366546 & -3.11214399655147 & -1.44972100103070 \\
\hline-3.08094129301026 & 2.51993869835353 & 3.38831841835419 \\
\hline-3.18293945655489 & -0.06843226268511 & 4.72720630809370 \\
\hline-3.40328441848694 & 4.50656261115694 & 5.52075599930602 \\
\hline-5.61243219267224 & 2.75110223641357 & 1.94538798362102 \\
\hline 1.92980414404131 & 3.88628525664639 & 3. 29191456028742 \\
\hline 4.31432398358138 & 3.99545404036624 & 1.60261059767044 \\
\hline 1.84759216719772 & 6.61087690295458 & 4.41095498531358 \\
\hline 2.46037437646116 & 2.15331856272516 & 5.57661828604699 \\
\hline 0.42658398359208 & -12.37569944847077 & 4.40436609657625 \\
\hline 3.14687845063586 & -3.19109715959191 & -1.07049600691105 \\
\hline 5.20547367988788 & -2.33858931355467 & 2.26095226292194 \\
\hline 6.60828918807538 & -3.74944199194382 & 1.70758711463261 \\
\hline 5.92705354930467 & -0.49757891840078 & 1.72665324691282 \\
\hline 5.03383400589550 & -2.42355974897267 & 4.30859928946709 \\
\hline 4.35485370009203 & 2.54055222768279 & 6.30198589244881 \\
\hline 1.15562867975521 & 2.50376819692917 & 7.12641569709893 \\
\hline 2.35316887454275 & 0.16971142589045 & 5.10888399655208 \\
\hline-7.13784387510580 & 2.25158056446502 & 3. 24216140046876 \\
\hline-5.98116787461458 & 4.67287954964555 & 1.30982421969164 \\
\hline-5.76633181792867 & 1.48837658440051 & 0.34834691548189 \\
\hline 3.78990727136045 & -2.44191161262339 & -4.68865230563359 \\
\hline 5.42698255536470 & 0.15279323912460 & -5.90759863167442 \\
\hline 4.81770090156437 & 0.00964673590853 & -2.66150933607300 \\
\hline-2.82735689396460 & -3.67667995949731 & 0.18933227240804 \\
\hline-5.30087146094011 & -1.71595006786651 & -0.87375803307526 \\
\hline-4.97233167375281 & -4.75562383781593 & -2.10963261029118 \\
\hline 4.43755314687532 & 2.43212933327885 & 0.29784891477856 \\
\hline
\end{tabular}




$\begin{array}{rrrr}4.37331652861104 & 5.73378363230268 & 0.49628306939027 & \mathrm{~h} \\ 5.99601320765826 & 4.01269751646399 & 2.79742389748830 & \mathrm{~h} \\ -3.38847932719643 & 6.44689784070664 & 4.83304765077999 & \mathrm{~h} \\ -5.25280808085060 & 4.19736784119047 & 6.38321855032648 & \mathrm{~h} \\ -2.02211791424617 & 4.33055516502466 & 7.02688679022193 & \mathrm{~h} \\ 0.99436963527745 & 5.16071348101838 & -5.70421824113963 & \mathrm{~h} \\ 2.91780724316631 & 4.51496665888463 & -3.04707464946739 & \mathrm{~h} \\ 4.09753723964382 & 4.10367489507758 & -6.10704181125725 & \mathrm{~h} \\ -4.80002039709205 & -3.86125129248652 & -6.30809394690421 & \mathrm{~h} \\ -5.75218941953492 & -0.81614684092413 & -5.46931350254750 & \mathrm{~h} \\ -3.27355583301826 & -1.25131639524742 & -7.63921373615564 & \mathrm{~h} \\ 3.70192007393468 & 6.96877872412889 & 5.24841618243520 & \mathrm{~h} \\ 1.56156127589144 & 8.06446367728600 & 2.98372746691623 & \mathrm{~h} \\ 0.47085937360131 & 6.88744234480252 & 5.89954927079962 & \mathrm{~h} \\ -0.72037736614716 & 1.75019841129341 & -8.43436415684407 & \mathrm{~h} \\ 2.51096354668518 & 1.29623031994400 & -8.95313869602940 & \mathrm{~h} \\ 0.59140317015033 & -1.31339669435456 & -8.30718709615194 & \mathrm{~h} \\ -3.39165952026299 & -1.61583035444042 & 3.40550091686800 & \mathrm{~h} \\ -1.53623093026581 & -0.43331848855517 & 5.89203149372727 & \mathrm{~h} \\ -4.83213401408998 & -0.07981414552213 & 5.97435537804107 & \mathrm{~h} \\ -1.75242556146852 & -6.09016463796319 & -4.64435127905798 & \mathrm{~h} \\ 0.76566816034777 & -4.99759284742265 & -2.85002399704984 & \mathrm{~h} \\ 0.53598824475596 & -4.18552277399751 & -6.06949291141109 & \mathrm{~h} \\ - & - & \end{array}$

